\title{
Functional recovery of odor representations in regenerated sensory inputs to the olfactory bulb
}

\author{
Man C. Cheung ${ }^{1}$, Woochan Jang ${ }^{2}$, James E. Schwob ${ }^{2}$ and Matt Wachowiak ${ }^{1,3}$ * \\ ' Department of Biology, Boston University, Boston, MA, USA \\ ${ }^{2}$ Department of Anatomy and Cellular Biology, Tufts University School of Medicine, Boston, MA, USA \\ ${ }^{3}$ Brain Institute and Department of Neurobiology and Anatomy, University of Utah, Salt Lake City, UT, USA
}

\section{Edited by:}

Charles F. Stevens, The Salk Institute for Biological Studies, USA

\section{Reviewed by:}

Naoshige Uchida, Harvard University, USA

Kazushige Touhara, University of Tokyo, Japan

\section{*Correspondence:}

Matt Wachowiak, Brain Institute and Department of Neurobiology and Anatomy, University of Utah, 33 S. Wasatch Drive, Salt Lake City, UT 84112, USA

e-mail:matt.wachowiak@utah.edu
The olfactory system has a unique capacity for recovery from peripheral damage. After injury to the olfactory epithelium (OE), olfactory sensory neurons (OSNs) regenerate and re-converge on target glomeruli of the olfactory bulb (OB). Thus far, this process has been described anatomically for only a few defined populations of OSNs. Here we characterize this regeneration at a functional level by assessing how odor representations carried by OSN inputs to the OB recover after massive loss and regeneration of the sensory neuron population. We used chronic imaging of mice expressing synaptopHluorin in OSNs to monitor odor representations in the dorsal OB before lesion by the olfactotoxin methyl bromide and after a 12 week recovery period. Methyl bromide eliminated functional inputs to the $O B$, and these inputs recovered to near-normal levels of response magnitude within 12 weeks. We also found that the functional topography of odor representations recovered after lesion, with odorants evoking OSN input to glomerular foci within the same functional domains as before lesion. At a finer spatial scale, however, we found evidence for mistargeting of regenerated OSN axons onto OB targets, with odorants evoking synaptopHluorin signals in small foci that did not conform to a typical glomerular structure but whose distribution was nonetheless odorant-specific. These results indicate that OSNs have a robust ability to reestablish functional inputs to the OB and that the mechanisms underlying the topography of bulbar reinnervation during development persist in the adult and allow primary sensory representations to be largely restored after massive sensory neuron loss.

Keywords: olfactory bulb, regeneration, sensory neurons, synaptopHluorin, axon targeting

\section{INTRODUCTION}

The mammalian olfactory system has a remarkable capacity for regeneration of its primary sensory neurons (olfactory sensory neurons; OSNs) after loss due to injury, infection, or exposure to toxins. Even after a virtually complete loss of all OSNs, the population is restored to a level nearly indistinguishable from the original in terms of neuronal number and topography of odorant receptor (OR) protein expression (Schwob et al., 1999; Iwema et al., 2004). These newly-generated OSNs must reestablish convergent axonal connections with their appropriate targets in the olfactory bulb (OB). During normal development, the axons of all of the several thousand OSNs expressing the same OR converge onto just a few (2-4) of the $\sim 1600$ glomeruli in the OB (Mombaerts et al., 1996). Because individual glomeruli serve as functional units in the central coding and processing of odor information, reestablishing appropriate connections between OSNs and glomeruli is likely critical for normal olfactory function. For example, errors in the reinnervation of glomeruli may underlie olfactory dysfunction in humans recovering from olfactory loss due to trauma or infection (Doty, 1979; Meisami et al., 1998). More generally, reestablishing appropriate neural connectivity is a prerequisite for the full recovery of function of any sensory or motor system.
Previous studies have demonstrated that, in the adult, the targeting of OSN axons to glomeruli after lesion is subject to errors that do not occur during development (Schwob et al., 1999; Costanzo, 2000; St. John and Key, 2003; McMillan Carr et al., 2004; Blanco-Hernández et al., 2012). These errors include a lack of exclusive convergence of OSNs onto the same glomerulus and projection of at least some axons of a given OSN population to multiple, dispersed glomeruli (Costanzo, 2000; St. John and Key, 2003; Blanco-Hernández et al., 2012). The impact of this mistargeting on odor representations remains largely unclear, however: targeting has been examined for only three OR-defined group of OSNs out of the approximately 1000 ORs expressed in the rodent olfactory system (Gogos et al., 2000; St. John and Key, 2003; Blanco-Hernández et al., 2012). Thus there is no generalized picture of the effect of OSN loss and regeneration on functional odor representations in the CNS.

To address this question, we used mice expressing synaptopHluorin in OSNs (Bozza et al., 2004) to functionally assess how odor representations recover after lesioning the olfactory epithelium (OE) with the olfactotoxin methyl bromide (Schwob et al., 1999). We found that despite apparent errors in the exclusive convergence of OSNs onto glomeruli, odor representations involving multiple glomeruli largely recovered after lesion, with 
a topographic organization and overall magnitude similar to that seen before lesion. These results indicate that the olfactory system shows a robust capacity to regenerate functional inputs to the CNS in a manner that, in all but the most severe cases, preserves the broad spatial organization of odor representations that was present before injury. Thus, primary representations of odor information can be largely reconstituted in the adult even after large-scale neuronal loss, an ability unique among sensory systems.

\section{MATERIALS AND METHODS ANIMAL STRAINS AND CARE}

We used olfactory marker protein-synaptopHluorin (OMP-spH) mice (Bozza et al., 2004) that had been backcrossed into the C57/Bl6 strain and bred with the 129/SvImJ strain to generate first generation (F1) hemizygous mice. Only males were used in the study due to their sensitivity to methyl bromide. The F1 animals were housed under standard conditions in ventilated racks until 12 weeks of age before being exposed to MeBr. All mouse colonies were bred and maintained at the Boston University animal care facility. Animals were transported to Tufts University School of Medicine for exposure and were returned to Boston University on the same day. All surgical and experimental procedures were approved by the Boston University and Tufts University Institutional Animal Care and Use Committees.

\section{SURGERY}

Seven to ten days before lesioning, custom made head caps were implanted on the skull using aseptic surgical procedures. The head cap consisted of a custom-milled aluminum plate that fit the skull snugly and to which two M2 bolts were attached. Animals were anesthetized with isoflurane, placed in a stereotaxic holder and the head cap was placed with its anterior edge aligned to the coronal suture and attached to the skull using dental acrylic. A piece of 30 gauge metal tubing was embedded in the dental acrylic posterior to the $\mathrm{OB}$ to serve as a fiducial marker for chronic imaging experiments. The acrylic, which was darkened to reduce autofluorescence, was extended from the head cap to the frontalnasal fissure, forming a well surrounding the dorsal OB.

For 3 days after surgery, wound margins were treated topically with the anesthetic bupivacaine $(1 \mathrm{mg} / \mathrm{ml}$, Sigma-Aldrich, St. Louis, MO) and cleaned with Betadine. Animals were also injected with the nonsteroidal anti-inflammatory carprofen $(5 \mathrm{mg} / \mathrm{kg} \mathrm{SC}$, Pfizer, New York, NY) and the antibiotic Baytril (3 mg/kg, IM). Animals were monitored closely for a 2 week span following surgery, the first imaging session, and $\mathrm{MeBr}$ exposure. Animals were given carprofen and Baytril as necessary.

\section{CHRONIC AND ACUTE OPTICAL IMAGING}

Animals outfitted with head caps were imaged at a minimum of three time points: a "pre-exposure" session to obtain baseline odor response maps, a "post-exposure" session 4 days after exposure to determine whether $\mathrm{MeBr}$ successfully induced lesions, and a final "recovery" session 12-13 weeks after exposure to determine the extent of regeneration and recovery of functional responses.
Immediately prior to pre-exposure imaging (minimum 3 days before $\mathrm{MeBr}$ exposure), animals were anesthetized with isoflurane and placed in a custom head holder mounted on goniometers and $x$ - and $y$-translation stages that allowed for independent positioning and rotation of the animal. The bone over the OBs was thinned to transparency, sparing a thin wall of dental acrylic surrounding the $\mathrm{OB}$ to form a well around the imaging window. After the first imaging session, the bone was dried and a layer of ethyl 2-cyanoacrylate glue (Instant Krazy Glue, KG925, Elmer's Products, Inc., Columbus, OH) was applied to the window. After the glue had set, the well was filled with silicone sealant (Kwik-Sil or Kwik-Cast, World Precision Instruments, Sarasota, FL). In nearly all cases, the combination of ethyl cyanoacrylate and silicone based adhesive preserved window translucency for approximately 1 month. There was more variability in preservation of window quality for the $\sim 14$ week period of the study. When windows were no longer translucent at the recovery time-point, the bone was re-thinned prior to imaging.

$\mathrm{OB}$ alignment across repeated imaging sessions was performed using one of two systems. For the first system, the platform position was fixed relative to a custom objective mount using an alignment bar. The micrometer translation stages allowed for adjustments relative to the initial fixation point. For the second method, a fiducial marker was embedded in the dental cement of the head cap. During the first imaging session, an image of the fiducial marker was taken for later alignment. The marker was placed so that it was visible in the field of view when the OB was centered under the objective.

For acute imaging sessions, anesthetized animals were placed on the custom head holder and the bone over the OB was thinned to transparency and a coverslip and mouse Ringer's solution placed over the OBs.

\section{OLFACTOMETRY}

Odorants were selected and delivered using a 16 channel computer-controlled olfactometer, as described previously (McGann et al., 2005). Odorant concentration across imaging sessions was confirmed before each session using a portable photoionization detector (miniRAE 2000, RAE Systems, San Jose, CA). All olfactometer parts (including the odorant chambers and anesthesia mask) were made from Teflon or PTFE tubing. Isoflurane was used as an anesthetic to maximize survival across multiple imaging sessions. Isoflurane and odorant were delivered to the animal through a custom anesthesia/odorant delivery mask that fit tightly around the mouse's snout. Isoflurane was vaporized (EZ-155, Euthanex Corp, Palmer, PA) and mixed with medical grade oxygen. To maintain constant oxygenation levels throughout the experiment, a solenoid was used to pass odorized air into the gas mask during odor presentation and filtered air between trials. The filtered air was set to match the flow rate of the odor line. Thus, the total flow was maintained at $0.9 \mathrm{~L} / \mathrm{min}$ during and between odor presentation $(0.5 \mathrm{~L} / \mathrm{min}$ isoflurane and oxygen with either $0.4 \mathrm{~L} / \mathrm{min}$ air or odorized air). In acute experiments, we used a conventional concentric delivery nozzle, described previously (Lam et al., 2000). In this case, total air flow was $0.5 \mathrm{~L} / \mathrm{min}$. 
Odorants used (and their purities) included 2-hexanone (98\%), 2-butanone (99.5\%), ethyl butyrate (99\%), methyl valerate (99\%), trans-2-methyl-2-butenal (96\%), isovaleraldehyde (97\%), 2-aminoacetophenone (98\%), hexyl acetate (99\%), benzaldehyde (99\%), phenylacetaldehyde (90\%), and methyl salicylate (99\%) from Sigma-Aldrich; butyric acid (99.5\%) and butyl acetate (99.5\%) from MP Biomedicals Inc.; and eugenol (99\%), menthone $(97 \%)$, acetophenone (98\%) and methyl benzoate (98\%) from Fluka.

\section{METHYL BROMIDE LESION}

Animals were exposed to MeBr as previously described (Schwob et al., 1995, 1999; Chen et al., 2004). F1 OMP-spH heterozygous $(\mathrm{C} 57 / \mathrm{Bl6} \times 129 \mathrm{SvImJ})$ males were exposed unilaterally to $\mathrm{MeBr}$ at 12 weeks of age. One side was protected by insertion of a plug made from polyethylene tubing and suture (Cummings et al., 1997) and sealed at the external naris with superglue. Animals were placed into a Plexiglas chamber and exposed to $\mathrm{MeBr}$ gas (Matheson Gas Products, East Rutherford, NJ). MeBr was diluted into purified air (210-240 ppm), with total flow at $10 \mathrm{~L} / \mathrm{min}$ and length of exposure set at 6 or $8 \mathrm{~h}$. Nose plugs were removed the following day.

\section{DATA ACQUISITION AND ANALYSIS}

Optical signals from the dorsal OB were acquired with standard wide-field epifluorescence microscopy as described previously (Bozza et al., 2004). Epifluorescence imaging was performed using an Olympus BX51 illumination turret with a 150-W Xenon arc lamp (Opti-Quip, Highland Mills, NY) at 50\% intensity (attenuated with an ND50 filter), with the following fluorescence filter set from Chroma Technology (Rockingham, VT): HQ480/40 (exciter), Q505LP (dichroic), HQ535/50 (emitter), with either a $4 X$ (0.28 N.A.) air or $20 X$ (0.95 N.A.) water immersion objective. Odorant-evoked signals were recorded and digitized at 14-bit resolution using a back-illuminated CCD camera (NeuroCCD, SM256; RedShirtImaging, Decatur, GA) at $256 \times 256$ pixel resolution and a frame rate of $7 \mathrm{~Hz}$. Data acquisition was performed with Neuroplex software (RedShirtImaging).

For display in the figures, odorant-evoked response maps were spatially low-pass filtered using a Gaussian kernel (sigma values given in text) and displayed, unless where noted, with fluorescence normalized to $95 \%$ of the maximum value of that map. In order to compare maps across imaging sessions in chronically imaged animals, image registration was performed by maximizing the correlation between resting fluorescence images or, when possible, using implanted fiducial markers (see above). In acutely imaged animals, OB positions were roughly aligned using the resting fluorescence image and the midline and posterior sinus as landmarks (Wachowiak and Cohen, 2001; Bozza et al., 2004). For calculating response amplitudes and positions of input to glomeruli, regions of interest (ROIs) were defined for all $\mathrm{spH}$ foci in the response maps using criteria based on focus size, signal-tonoise ratio and optical signal time-course to identify presumptive activated glomeruli (Wachowiak and Cohen, 2001; Bozza et al., 2004).

Consensus response map topographies (e.g., Figure 4C) were generated as described in Wachowiak et al. (2013). Briefly, individual response maps were aligned relative to the midline and caudal sinus, normalized to their own maxima, thresholded at $50 \%$, summed together, then smoothed with a $6 \times 6$ pixel mean kernel and the resulting maps renormalized and displayed as in Figure 4C. For statistical comparison of response map topographies (e.g., Figures 4D, E), maps were smoothed with a $3 \times 3$ pixel kernel, thresholded to include the top $70 \%$ of responses and centroids for each individual response map calculated from the mean of the positions of thresholded pixels. For comparison of centroid positions across animals, $x$ - and $y$-positions were mapped to the zero point defined by the intersection of the sagittal midline and the anterior limit of the caudal sinus (Wachowiak and Cohen, 2001; Bozza et al., 2004). For determining domain separation, the sum of the $x$-position squared and the $y$-position squared (the squared displacement) was used. For the calculation of foci diameter (e.g., Figure 5), response maps were first slightly smoothed with a low-pass filter (Gaussian kernel, $\sigma=10 \mu \mathrm{m}$ ) to remove noise and odorant-evoked foci chosen for analysis based on their signal-to-noise ratio and time-course of the odorantevoked fluorescence change. Focus sizes were measured by fitting the amplitude profiles of each ROI at perpendicular axes across each focus and taking the full-width at half-maximum (FWHM) of the fit along each axis. FWHM values for each axis were averaged to obtain a size value for each focus. To construct consensus odorant response maps for focus size analysis we projected individual, normalized response maps onto a single image using the maximal projection algorithm for confocal $z$ stacks (ImageJ).

\section{TWO-PHOTON IMAGING AND ANALYSIS}

All animals undergoing 2-photon laser scanning microscopy (2PLSM) at the terminal imaging session were anesthetized with pentobarbital before removal of the bone over the OB. The dura was also removed and agarose (1.2\% in mouse Ringer's) was placed onto the $\mathrm{OB}$ and coverslipped; petroleum jelly was used to seal the cranial window. Imaging was performed on a custom microscope that allowed for wide-field epifluorescence or multiphoton imaging through the same objective (20X, 0.95 N.A., water immersion; Olympus, Melville, NY). A 150-W Xenon arc lamp provided wide-field illumination at $2.8-6 \%$ of full intensity through the same filter set as described above. Two-photon fluorescence was excited by a mode-locked Ti:Sapphire laser (SpectraPhysics, 150 fs, $76 \mathrm{MHz}$; pumped by a 5W Millenia Vs. laser); emitted light was reflected through a mirror placed at the back aperture of the objective and directed to a bialkali photomultiplier (HC125-02, Hamamatsu Corporation, Bridgewater, NJ) fitted with an emission filter (Omega Optical, 535/45). Image acquisition was controlled by custom software in LabView (developed by Dr. J. Mertz, Boston University). For imaging odorant-evoked responses, acquisition rate was $8 \mathrm{~Hz}$ with a pixel resolution of $1.6 \mu \mathrm{m}$. Response maps obtained with 2PLSM were averaged from 5 to 10 trials to improve signal-to-noise ratio. Relative fluorescence changes were calculated using the eight frames before odorant onset as the baseline fluorescence and an average of eight frames at the peak of the evoked signal as the response. ROIs were defined using resting multiphoton resting fluorescence images. 


\section{CONFOCAL MICROSCOPY AND HISTOLOGY}

Following the terminal imaging session, animals were overdosed with pentobarbital and perfused intracardially with mouse Ringer's solution $(20 \mathrm{ml})$, followed by cold $4 \%$ paraformaldehyde (20 ml, 0.05 M PBS, pH 7.0). For confocal imaging, the bone surrounding the OBs was removed under alkaline PBS $(0.1 \mathrm{M}$, $\mathrm{pH}$ 7.9) and the OBs were scanned in situ with a confocal microscope (LSM 510, Carl Zeiss MicroImaging Inc., Thornwood, NY) to assess OSN innervation of glomeruli using OMP$\mathrm{spH}$ fluorescence. The $\mathrm{OE}$ and $\mathrm{OB}$ were then preserved in $4 \%$ paraformaldehyde until cryoprotection. Coronal sections of the olfactory tissue from the OE to $\mathrm{OB}$ were prepared using a cryostat at $50 \mu \mathrm{m} /$ section. Frozen sections were counterstained with cresyl violet and mounted.

\section{RESULTS}

We used the gaseous olfactotoxin $\mathrm{MeBr}$ to unilaterally lesion the $\mathrm{OE}$ of mice expressing $\mathrm{spH}$, an optical reporter of transmitter release, in all OSNs (OMP-spH mice; Bozza et al., 2004). Male, hemizygous OMP-spH mice were used in all experiments and lesioned at 12 weeks of age (see Section Materials and Methods). In all experiments one naris was protected from $\mathrm{MeBr}$ exposure with a plug that was removed after the exposure period (Cummings et al., 1997). Exposure to $\mathrm{MeBr}$ gas has been used extensively to lesion the $\mathrm{OE}$ of rats, and the severity of lesion can be controlled by varying $\mathrm{MeBr}$ concentration and duration of exposure (Schwob et al., 1995, 1999; Iwema et al., 2004). The time-course and cellular changes underlying degeneration and subsequent recovery of the $\mathrm{OE}$ after $\mathrm{MeBr}$ exposure have also been well-characterized (Schwob et al., 1999). Our goal was to assess the degree to which OSNs regenerate functional connections to glomeruli of the $\mathrm{OB}$, where the central representations of odor information are initially formed. The general approach was to compare glomerular odor representations using spH-mediated optical signals (Bozza et al., 2004) before lesion and after a recovery period.

\section{LONG-TERM, CHRONIC IMAGING OF SENSORY INPUTS IN OLFACTORY MARKER PROTEIN-SYNAPTOPHLUORIN (OMP-spH) MICE}

It was first necessary to establish the stability of odorant representations over a time-period sufficient to allow for OSN recoveryat least 60 days (Schwob et al., 1999)_and under conditions that allow for repeated optical imaging in the same animal. We have previously shown that OSN inputs can be chronically imaged in OMP-spH mice and remain stable over at least 7 days (Bozza et al., 2004). Here, we extended this time-period. We installed a chronic imaging window over the dorsal OB (see Section Materials and Methods) and imaged odorant-evoked $\mathrm{spH}$ response maps in three OMP-spH mice over periods of 111, 124 and 174 days.

Figure 1 shows examples of $\mathrm{spH}$ response maps imaged at different time-points in three animals. In all three animals, response maps remained similar across this period. The most significant variability in maps arose from differences of up to $50 \%$ in relative signal magnitude in different glomeruli (highlighted by arrows, Figure 1A); these differences could affect the absolute number of glomeruli activated above an arbitrary threshold level. Such variability likely reflects differences in overall sensitivity in different imaging sessions, due (for example) to changes in nasal patency (Oka et al., 2009), experience-dependent plasticity (Jones et al., 2008; Kass et al., 2013) or modulatory influences (McGann et al., 2005; Pírez and Wachowiak, 2008). Nonetheless the approximate number and relative position of activated glomeruli remained consistent across imaging sessions (Figures 1B, C), indicating that the procedures involved in chronic imaging (head cap and imaging window implantation, repeated anesthesia sessions and odorant presentations) did not induce apparent changes in functional connections between OSNs and their target glomeruli.

To assess the precision with which response maps could be monitored across time-points, we compared the positions of the few (2-4) most strongly-activated glomeruli in maps evoked by the same odorant in different sessions. OB images were aligned as described in the Section Materials and Methods, then the distance between each glomerulus at the initial time-point and its nearest neighbor at the later time-point was measured. Using this measure, the average change in the position of $\mathrm{spH}$ signal foci between baseline and the later time-point (111-176 days) was $71.0 \pm 29.1 \mu \mathrm{m}$ (mean \pm s.d.; $n=21$ glomeruli taken from three animals and using nine odorants). Thus, in unlesioned animals, we are able to chronically map functional inputs to glomeruli with a spatial precision of smaller than the average diameter of a glomerulus (Bozza et al., 2004).

\section{MeBr EXPOSURE ELIMINATES ODORANT-EVOKED RESPONSES IN THE OLFACTORY BULB (OB)}

$\mathrm{MeBr}$ potency has not been as extensively characterized in mice (Chen et al., 2004) as it has in rats (Schwob et al., 1995, 1999; Iwema et al., 2004); in addition, the relationship between the initial loss of OSNs and functional inputs to the OB immediately after $\mathrm{MeBr}$ exposure is unclear. Thus we next examined the effect of $\mathrm{MeBr}$ exposure on odorant-evoked $\mathrm{spH}$ signals and on OSN loss. In these animals spH signals were imaged shortly (4-10 days) after lesion, after which the mouse was sacrificed and damage to the $\mathrm{OE}$ assessed histologically. We used different $\mathrm{MeBr}$ exposure protocols that resulted in a range of lesion severity.

At a $\mathrm{MeBr}$ exposure of $215 \mathrm{ppm}$ for $8 \mathrm{~h}(n=10$ mice $)$ or $230 \mathrm{ppm}$ for $6 \mathrm{~h}(n=4)$, exposure resulted in a complete loss of detectable odorant-evoked $\mathrm{spH}$ signals in half (7/14) of all mice (Figure 2A). Higher dosages resulted in significant rates of mortality (not shown). In animals showing a loss of odorantevoked signals, resting $\mathrm{spH}$ fluorescence on the MeBr-exposed side was also diminished although not eliminated entirely at 4-10 days post-lesion (Figure 2A). Resting fluorescence and $\mathrm{spH}$ response maps remained robust on the protected side of all mice (Figure 2). In approximately $20 \%$ of MeBr-exposed animals (3 of 14), resting fluorescence and evoked $\mathrm{spH}$ signals were still detectable but weaker on the exposed side compared to the pre-lesion imaging session or the protected side (Figure $2 \mathbf{B}$, ii). Further quantification from similarly-lesioned mice in a different cohort is provided below. In the remaining approximately $30 \%$ of animals (4 of 14), resting fluorescence and $\mathrm{spH}$ signals were similar in magnitude to the pre-lesion session or to those on the protected side (Figure 2B, iii). These results indicate that $\mathrm{MeBr}$ exposure can eliminate OSN responsiveness unilaterally 
A

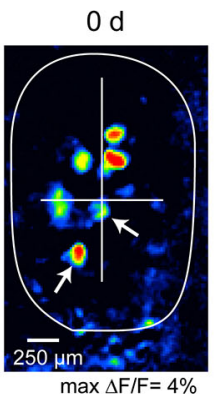

B

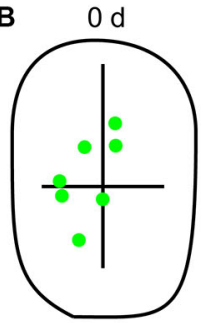

methyl valerate
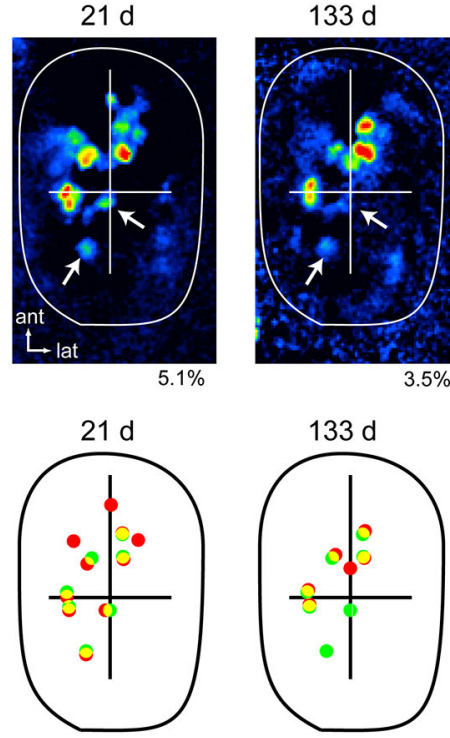

C

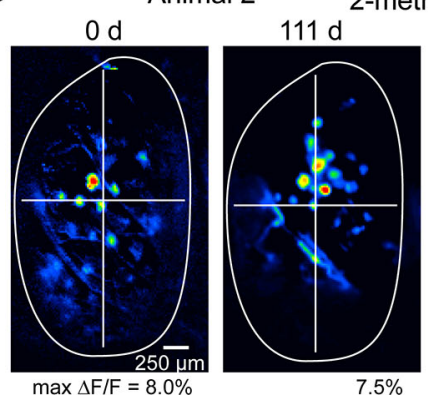

FIGURE 1 | Long-term stability of OSN representations during chronic imaging from the OB. (A) $\mathrm{spH}$ response maps evoked by the same odorant (methyl valerate, $1 \%$ s.v.) imaged at four time points in the same animal (expressed as days after initial imaging session). The approximate outline of the dorsal OB is shown; crossbars are for comparisons across maps. Each map is normalized to its own maximum; absolute maximum signal amplitudes ("max $\Delta F / F$ ") are given below each map. Arrows indicate signal foci that are apparent in each map but show reduced

and that the effectiveness of exposure is more variable than has been previously observed in rats (Schwob et al., 1995, 1999).

In this cohort, mice were sacrificed immediately after imaging and $\mathrm{MeBr}$-induced damage to the $\mathrm{OE}$ was assessed histologically. Acutely after exposure, damaged areas were easily evident in hematoxylin and eosin (H\&E)-stained sections by the sloughing of sustentacular cells and neurons, as previously described (Schwob et al., 1995). Mice showed some variation in the severity of the damage from animal to animal even when carefully controlled for age, weight and strain such that sparing was seen in some areas while in other areas damage was so severe that the basal lamina was breached leading to a serum exudate in the nasal cavity - a circumstance that precludes regeneration of the epithelium (Schwob et al., 1995). The portion of the OE that projects to the region of the dorsal $\mathrm{OB}$ imaged in these experiments corresponds roughly to the territory defined by lack
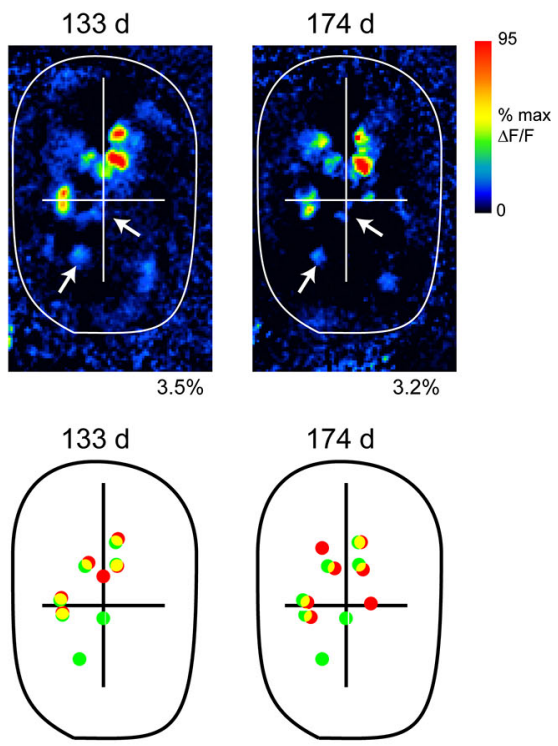

Animal 3

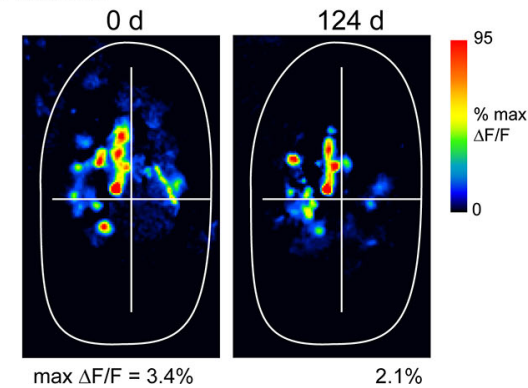

amplitudes over time. Maps were smoothed with a Gaussian kernel $(\sigma=25 \mu \mathrm{m}$, kernel width $=70 \mu \mathrm{m})$ for display. (B) Comparison of $\mathrm{spH}$ signal foci locations for responses at day 0 and each later time-point, taken from maps in (A). Dots identify signal foci with amplitudes above $30 \%$ of maximal amplitude. Green dots indicate foci in initial session, red indicates foci in the current session, yellow indicates colocalized foci. (C) $\mathrm{spH}$ response maps for two additional animals imaged at 111 and 124 days time-points; odorant: 2-methyl 2-butenal. of staining with anti-OCAM/mamFasII antibodies (Schwob and Gottlieb, 1986; Uchida et al., 2000); thus further description of the OE after lesion recovery (see below) is limited to that area.

Mice showing a complete loss of odorant-evoked $\mathrm{spH}$ signals were characterized by complete or near complete destruction of the neuronal and sustentacular cell populations in the dorsal half of the OE (Figure 2B, i). In these cases, the full extent of the dorsal OE was damaged based on comparison with the protected side, and across the vast majority of that epithelium the neuronal population was destroyed completely (see "exposed" side of OE image, Figure 2B, i). Mice showing weakened $\mathrm{spH}$ signals and classified as having intermediate functional lesion (Figure 2B, ii) also showed significant damage to the OE, but substantial areas of the dorsal epithelium were spared, particularly at posterior levels of the nasal cavity. Surprisingly, even those mice that retained robust $\mathrm{spH}$ signals and so were classified as having 

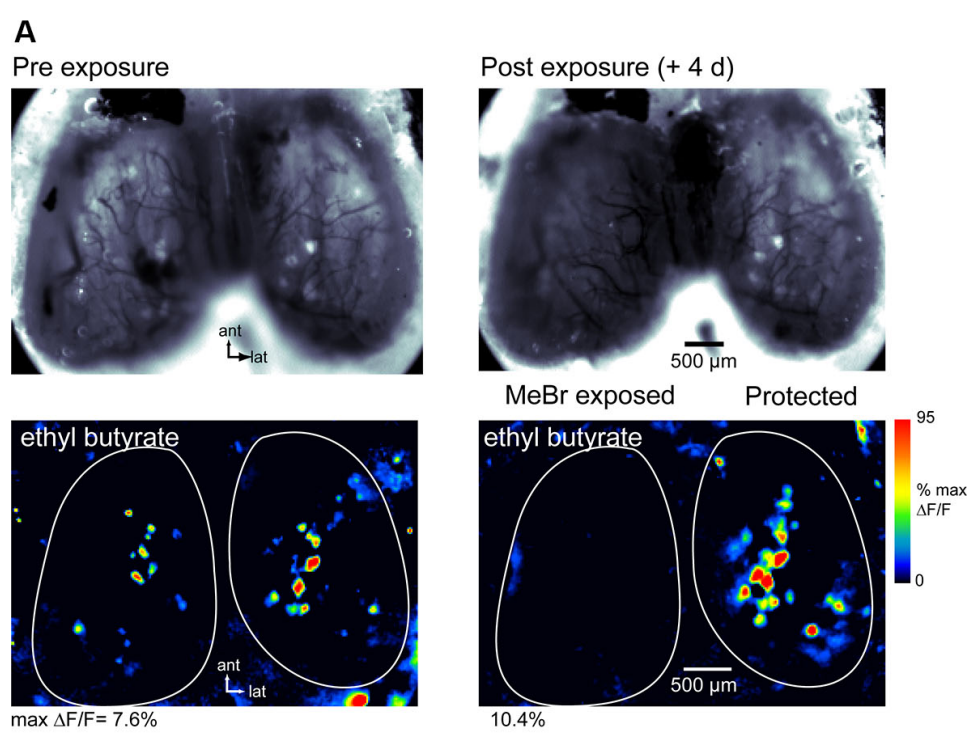

B

Post exposure (+4 - 10 d)
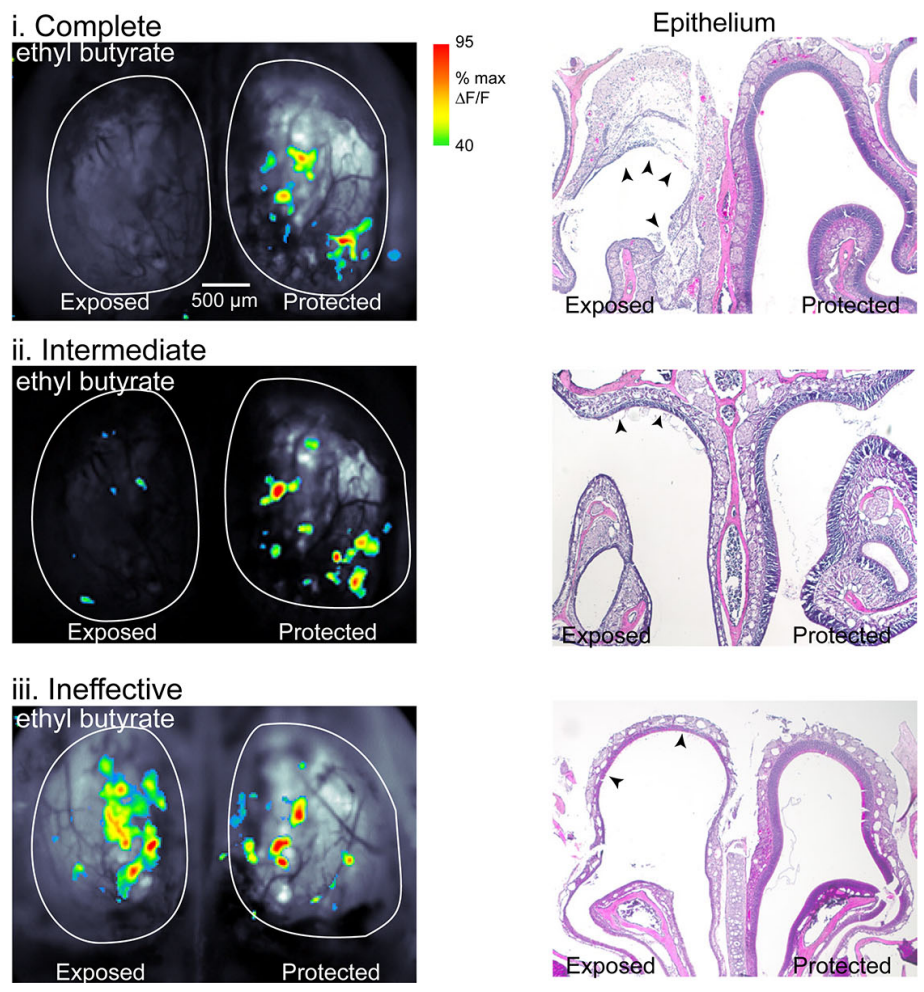

FIGURE 2 | Effect of MeBr lesion on functional OSN inputs to the OB. (A) Resting fluorescence images (top) and response maps (bottom) imaged in the same animal 10 days before (left) and 4 days after (right) unilateral $\mathrm{MeBr}$ exposure. This animal showed a decrease in resting fluorescence and a complete loss of odorant-evoked $\mathrm{spH}$ signals on the exposed side. Responses on the protected side were unaffected. Odorant, ethyl butyrate (1\% s.v.). (B) Response maps overlaid on resting fluorescence images (left column) and post-hoc H\&E-stained nasal cavity sections ("Epithelium", right column) for three additional animals showing different degrees of functional loss after $\mathrm{MeBr}$ exposure. Effects were classified as complete (i),

intermediate (ii) or ineffective (iii) based on the amplitude and sensitivity of the odorant-evoked spH signal on the MeBr-exposed side (See the text). Response maps scaled as in (A) but thresholded at $40 \%$ of maximal $\delta \mathrm{F} / \mathrm{F}$.
The nasal cavity showed widespread damage to the OE in all three animals. In the complete lesion (i), the full tangential extent of the dorsomedial epithelium has been lesioned and in many areas the damage extends through the basal lamina (arrowheads), leading to an exudation that will become organized as endonasal scar tissue (cf. asterisks in Figure 8B). In the intermediate lesion (ii), the full tangential extent of the dorsomedial epithelium is damaged but there are some residual neurons along the roof of the dorsal meatus (arrowheads). In the "ineffective" lesion (iii), the full tangential extent of the dorsomedial epithelium is also damaged but there are residual neurons along the roof of the meatus in the area between the two arrowheads. Resting spH fluorescence in the OB is sharply diminished in the "complete" and "intermediate" lesions, but appears normal in the "ineffective" lesion. 
ineffective functional lesion showed at least moderate OE damage, particularly in the far anterior and far posterior nasal cavity (Figure 2B, iii). Overall, these results indicate that functional imaging of odorant-evoked $\mathrm{spH}$ signals is a stringent assay for the effectiveness of MeBr lesion: animals showing a complete loss of $\mathrm{spH}$ signal likely have only a small fraction of OSNs surviving after lesion, with larger survival rates reflected in robust odorantevoked signals.

\section{FUNCTIONAL INPUTS TO OLFACTORY BULB (OB) GLOMERULI RECOVER AFTER MeBr LESION}

To assess the functional recovery of OSN connections to OB glomeruli after $\mathrm{MeBr}$ lesion, we imaged $\mathrm{spH}$ odorant response maps at three time-points: 4-10 days before lesion, approximately 4 days after lesion to assess lesion effectiveness, and 12-13 weeks after lesion to assess recovery. Ten mice were unilaterally exposed to $\mathrm{MeBr}$ using a dosage and exposure protocol (235 ppm for $6.5 \mathrm{~h}$ ) similar to that used to assess lesion effectiveness (above). Of these animals, four showed persistent odorant-evoked responses at the assessment session and so were excluded from further analysis; the remaining six animals showed complete loss of $\mathrm{spH}$ signals on the exposed side at assessment. In none of the mice did we observe any obvious behavioral changes either immediately after unilateral exposure or during the recovery period.

In all six of these mice, odorants evoked clear $\mathrm{spH}$ signals on the lesioned side at 12 weeks post-lesion. Figures 3 A, B show odorant response maps and $\mathrm{spH}$ signal traces in a representative animal. Evoked spH signals 12 weeks post-lesion appeared roughly similar to those observed before lesion, consisting of spatially heterogeneous responses with numerous discrete signal foci (Figure 3A). In many cases these signal foci appeared in locations that were nearly identical to those observed before lesion (Figure 3A, arrows). Using the ROIs determined at baseline imaging for both the exposed and protected OBs, we were able to identify and measure odorant-evoked $\mathrm{spH}$ signals after the 12 weeks recovery period. The time-course of the odorant-evoked $\mathrm{spH}$ signal was also similar before lesion and after recovery (Figure 3B). Across animals, the peak amplitude of the spH signal was similar before lesion and after recovery for these animals (prelesion: $2.6 \pm 1.5 \%$; mean \pm s.d.; recovery: $3.2 \pm 0.7 \%$; $p=0.45$, paired $t$-test, $n=22$ odorant pairs across six animals; Figure $3 \mathrm{C}$ ).

To address potential confounds of the chronic imaging window on OSN recovery (Xu et al., 2007), a separate set of four mice were exposed unilaterally to $\mathrm{MeBr}(215 \mathrm{ppm}$ for $8 \mathrm{~h}$ ) and $\mathrm{spH}$ signals imaged in a single session 12 weeks after exposure. Thus in these mice there was no baseline session or assessment of lesion effectiveness, but odorant-evoked $\mathrm{spH}$ response maps were compared between the exposed and protected sides. In these animals, response maps appeared qualitatively similar to those seen on the protected $\mathrm{OB}$, and included individual signal foci that were located in a position that was symmetric with foci on the unlesioned side (Figure 3D). Peak-amplitude $\mathrm{spH}$ signals were similar on the exposed ( $n=28$ glomeruli from four animals) and unexposed sides ( $n=32$ glomeruli, four animals; $p=0.35$, unpaired $t$-test). Thus, OSNs that are replaced after MeBr lesion reestablish convergent functional connections to glomeruli of the $\mathrm{OB}$.

\section{RECOVERY OF SENSORY INPUT MAP TOPOGRAPHY AFTER OLFACTORY SENSORY NEURON (OSN) REGENERATION}

Projections of OSNs to OB glomeruli show at least two levels of spatial organization: (1) OSNs expressing a given OR converge onto a single glomerulus whose position within a domain varies by several hundred microns in different animals and remains relatively constant in the same animal over time (Strotmann et al., 2000; Schaefer et al., 2001; Costanzo and Kobayashi, 2010; see also Figure 1); and (2) projections show a broad topography in which OSNs of a particular class project within spatial domains spanning large regions of the bulbar surface (Nagao et al., 2002; Bozza et al., 2009; Pacifico et al., 2012). Chronic imaging of odorant response maps before lesion and after recovery showed that $\mathrm{spH}$ signals in lesion-recovered animals often differed slightly in the precise location of individual signal foci, but that $\mathrm{spH}$ signals remained clustered in locations similar to those seen in pre-lesion response maps (Figures 4A, B). These examples suggest that, while precise targeting of OSNs to pre-existing glomerular locations may be disrupted in regenerated OSNs, projections to the $\mathrm{OB}$ may recover with sufficient precision to preserve the topography of functional domains related to particular odorants.

To analyze the topography of lesion-recovered OSN projections more thoroughly, we examined response maps for odorants that have been previously shown to preferentially activate glomeruli in either the anterior (ANT) dorsal OB (aliphatic aldehydes and acids and some esters) or the caudolateral (CL) dorsal OB (ketones and aromatics) (Uchida et al., 2000; Wachowiak and Cohen, 2001; Bozza et al., 2004, 2009; Takahashi et al., 2004; Matsumoto et al., 2010). Because different odorants were tested in different animals, response maps were pooled into either ANT-activating or CL-activating groups depending on odorant identity. ANT odorants were: ethyl butyrate, hexaldehyde, 2methyl-2-butenal, butyl acetate and butyric acid; CL odorants were: acetophenone, 2-hexanone, menthone, methyl benzoate and eugenol. For a qualitative comparison of response map topography before and after lesion recovery, we generated consensus topographies as described previously (Wachowiak et al., 2013) and in Section Materials and Methods, compiled from pre-lesion and lesion recovery imaging sessions in the same chronicallyimaged animals. ANT and CL odorants evoked the strongest responses in similar $\mathrm{OB}$ regions before lesion and after recovery (Figure 4C).

For quantitative comparison of response map topographies, maps from ANT and CL odorants and between pre-lesion (baseline) and recovery sessions were compared using the centroid of each response map (see Section Materials and Methods; Figure 4D). Centroid positions were compared statistically using a 4-factor ANOVA with the following factors: ANT-odorants at baseline; ANT-odorants at recovery; CL-odorants at baseline and CL-odorants at recovery. There were a minimum of six response maps (at least six different odorants) for each factor; $\mathrm{MeBr}$ exposed and protected sides were analyzed separately. In the pre-exposure (baseline) session, as expected, maps for ANT- or CL-activating odorants were located in largely non-overlapping domains in the ANT- or CL- OB, respectively (Figure 4D), with distinct centroid positions as determined by the 4 -factor ANOVA 


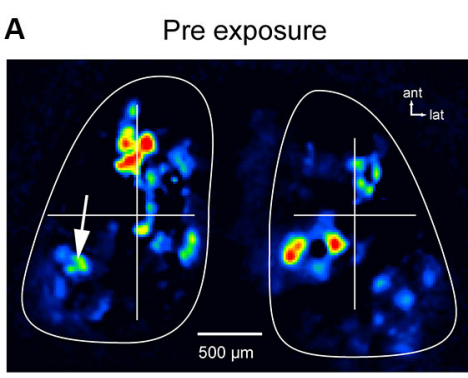

ethyl butyrate

B

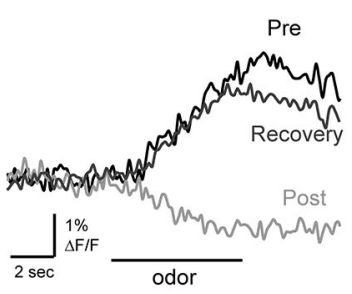

ii. response maps

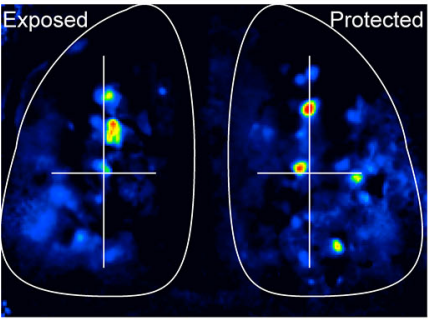

hexyl acetate

C

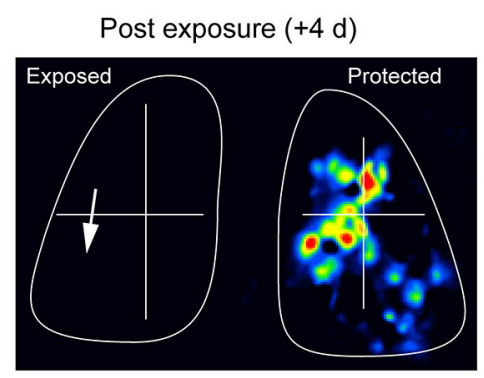

ethyl butyrate
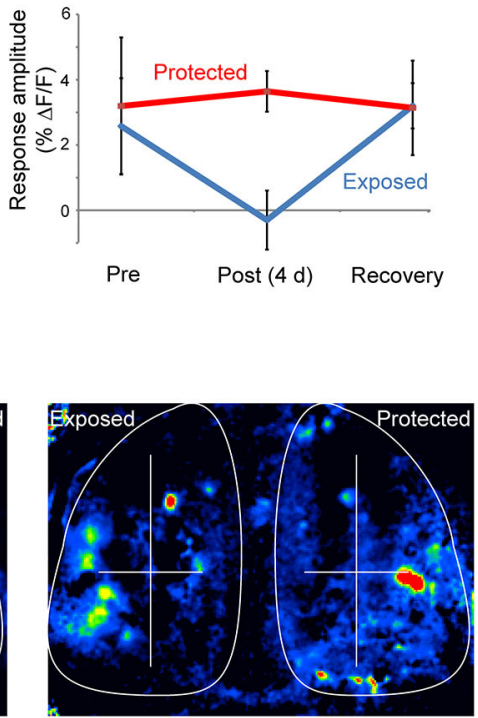

phenacetaldehyde

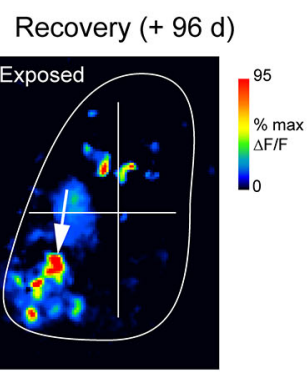

ethyl butyrate

D Acutely imaged (+ 84 d)
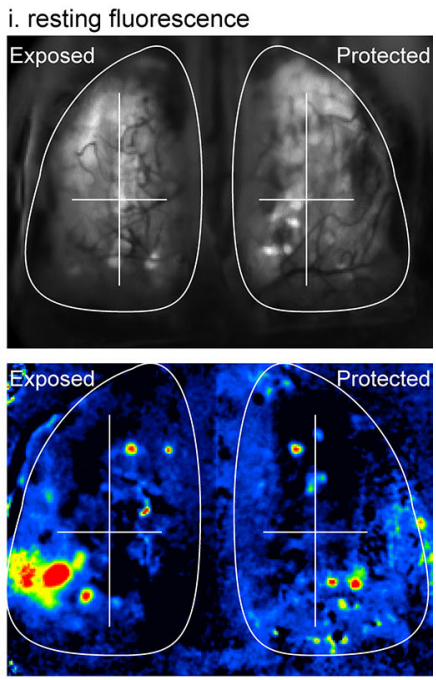

methyl salicylate
FIGURE 3 | Functional recovery of OSN inputs to the OB after MeBr lesion. (A) Resting fluorescence and response maps imaged in the same animal before ("Pre-exposure"), 4 days after ("Post-exposure") and 96 days after ("Recovery") unilateral exposure to MeBr. Odorant was ethyl butyrate in all cases. Odorant-evoked responses were eliminated post-exposure; responses and resting fluorescence (not shown) reappeared at Recovery. (B) Traces showing time-course of the odorant-evoked $\mathrm{spH}$ signal from one location (indicated by arrow in (A)), which was similar before lesion and after recovery. Fluorescence decrease at the "Post" exposure time-point reflects intrinsic hemodynamic artifacts described previously. (C) Summary data showing $\mathrm{spH}$ response amplitudes on the exposed and protected sides before lesion and after recovery. See the text for details. Error bars indicate s.d. (D) Odorant response maps imaged in a single acute session at 84 days post unilateral $\mathrm{MeBr}$ exposure. (i) Resting $\mathrm{spH}$ fluorescence. (ii) Odorant-evoked response maps evoked by three different odorants appear similar on the exposed and protected OBs, with the most variance appearing as different relative amplitudes of the $\mathrm{spH}$ signal. Note presence of putative homologous individual glomeruli on each side, especially in the anterior OB. To facilitate comparison, response maps from each side were normalized separately to their own maximum (same pseudocolor scale as in (A)).
$(F(3,49)=2.885, p<0.05)$ and a post-hoc test comparing ANT- and CL-odorant centroids at baseline (Fisher's exact test: $p<0.05)$. However, there was no significant change in ANT- or CL-odorant map topography after lesion recovery (Figure 4D), with post-hoc analyses reporting no difference in centroid locations between baseline and recovery sessions (Fisher's exact test; ANT: $p>0.34$; CL: $p>0.50$ ). In agreement with the results in chronically-imaged animals, in the four animals that were exposed to $\mathrm{MeBr}$ and acutely imaged at the recovery stage, ANT- and CL-odorants evoked inputs to regions with statistically distinct centroids (unpaired Student's $t$-test, ANT vs. CL positions, $n=23, p<0.001$ ), similar to those seen in the unexposed $\mathrm{OB}$ of the same animals (Figure $4 \mathrm{E}$ ). These results indicate that OSNs preferentially regenerate axonal projections to targets within their original functional domains on the $\mathrm{OB}$ surface, thus reconstituting the broad topography of glomerular activation that is a hallmark of primary odor representations in the OB.

\section{ATYPICAL CONVERGENCE OF OLFACTORY SENSORY NEURONS (OSNs) TO OLFACTORY BULB (OB) TARGETS AFTER LESION RECOVERY}

Close inspection of lesion-recovered response maps revealed numerous examples of $\mathrm{spH}$ signal foci that appeared smaller than a typical glomerulus. These smaller foci-or the presence of more diffuse spH signals_-could reflect OSN axons that failed to converge or that only partially innervated a glomerulus (St. John and Key, 2003; Blanco-Hernández et al., 2012). To examine these signals more carefully we imaged responses at highermagnification and smaller depth of field (20X, 0.95 N.A. objective, $3.5 \mu \mathrm{m}$ pixel resolution) using the same animals as in the above 
A

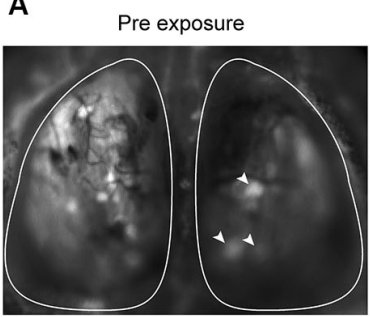

ethyl butyrate

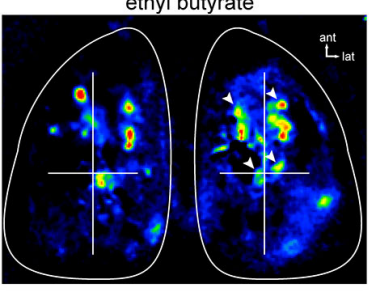

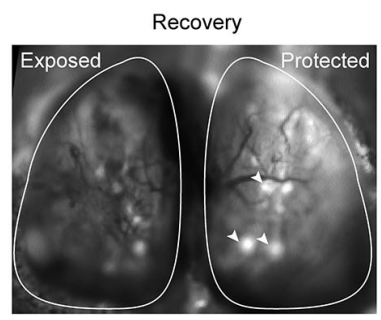

ethyl butyrate

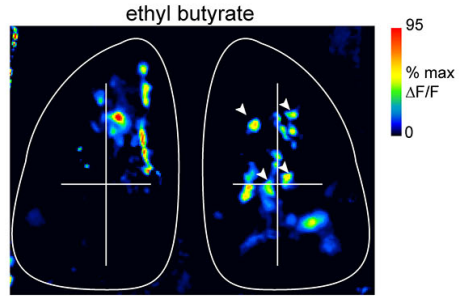

B
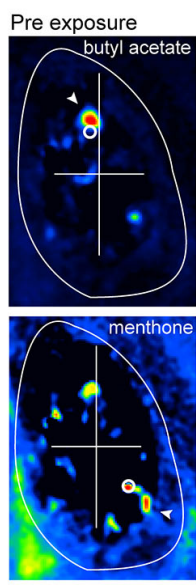
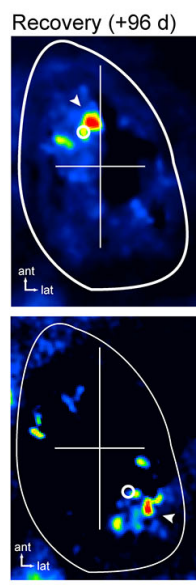

C

Consensus topographies

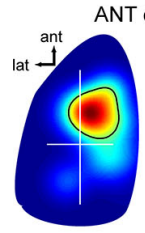

Pre

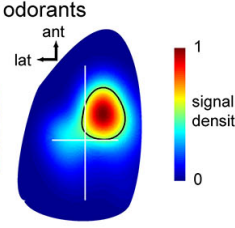

Recovery

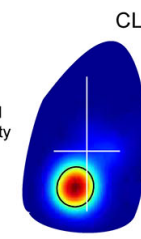

Pre
D

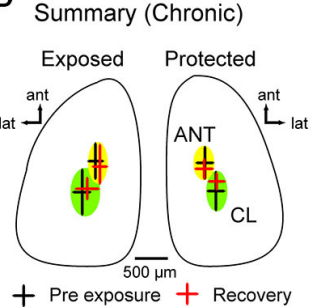

E Summary (Acute)

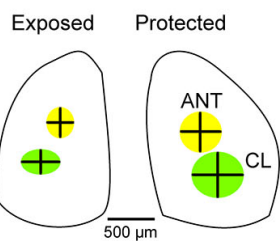

FIGURE 4 | Odor response map topography is reestablished after $\mathrm{MeBr}$ lesion and OSN recovery. (A) $\mathrm{spH}$ resting fluorescence and bilateral response maps imaged before unilateral $\mathrm{MeBr}$ exposure ("Pre-exposure") and after recovery in the same animal, shown for ethyl butyrate. On the protected side, blood vessel pattern and position of brightly fluorescent glomeruli is near-identical, as are locations of strongly-activated glomeruli (arrowheads). On the exposed side, brightly fluorescent glomeruli and $\mathrm{spH}$ responses are similar in amplitude and similar but not identical in location after recovery. (B) Additional examples showing spH response maps for two odorants that preferentially evoke input to the anterior (top) and caudal-lateral OB (bottom) respectively. For each odorant, response maps are topographically similar at both time-points. White circles indicate the location of the centroid of each map, calculated after smoothing and thresholding (See the text). Arrowheads indicates a particular $\mathrm{spH}$ focus (glomerulus) whose position is consistent in both pre-exposure and lesion-recovered maps. (C) Consensus topographies for anterior (ANT) and caudal-lateral (CL) odorant response maps compiled from chronically-imaged mice ( $n=5$ mice, 4-8 odorants per mouse) unilaterally exposed to $\mathrm{MeBr}$ and imaged before exposure ("Pre") and after lesion recovery ("Recovery"). Pseudocolor scale indicates relative density of odorant-evoked spH signal across all response maps in each category. Black contour indicates arbitrary $50 \%$ cutoff of relative density plot. See the text for analysis details. (D) Quantitative analysis of response topographies in chronically-imaged mice (same animals and odorants as in (C)). Crosshairs and shaded areas show centroid locations before lesion (black crosshairs, "Pre-exposure") and after recovery (red crosshairs, "Recovery") for anterior (ANT, yellow) and caudal-lateral (CL, green) odorants (See the text for list). The centers of the cross hairs denote average centroid location across all pooled odorants, with the arms and ellipses extending to 1 s.d. in $x$-and $y$-directions. Domains remained distinct for each time point and similar across time-points (note that this analysis differed slightly from that used to produce consensus topographies in (C); See the text for analysis details). (E) Centroid locations analyzed and plotted as in (D) for acutely-imaged animals, showing similar distribution of centroid locations for exposed and protected sides imaged in a single recovery session. analysis. Imaging at this resolution confirmed that in lesionrecovered mice, odorant-evoked $\mathrm{spH}$ signals often appeared in foci that were subglomerular in size (Figures 5A, B). Such foci were also apparent in acutely-imaged MeBr-treated animals (Figure 5A, iii), indicating that these were not a result of chronic window implantation. $\mathrm{spH}$ signals from subglomerular-sized foci displayed response dynamics that were similar to those from unexposed animals or larger foci (Figure 5C).

We quantitatively compared $\mathrm{spH}$ signal foci sizes in maps taken from baseline and lesion-recovered imaging sessions by fitting the signal intensity profile of discrete foci to a Gaussian and measuring the FWHM of the fit (see Section Materials and Methods and Meister and Bonhoeffer, 2001). These measurements were made in acutely-imaged, MeBr-exposed animals imaged at the recovery time-point. On the side exposed to $\mathrm{MeBr}$, there was a larger number of small-diameter foci (Figures 5D, E), leading to significant reduction in the mean focus size from $90.7 \pm 20.8 \mu \mathrm{m}$. ( $n=45$ glomeruli from three animals $)$ to $67.6 \pm 33.5 \mu \mathrm{m}(n=100$ glomeruli from four animals; $p<$ 0.0001 , unpaired $t$-test). Thus, OSN inputs to lesion-recovered OBs frequently converge onto structures smaller than the size of typical glomeruli.

To investigate the underlying anatomical structure of lesionrecovered OSN inputs to the $\mathrm{OB}$, we used confocal microscopy to scan the intact dorsal $\mathrm{OB}$ of imaged preparations (see Section Materials and Methods). In control animals and on 
A Composite maps

i. Pre exposure

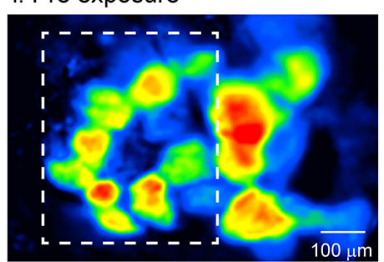

ii. Recovery (chronic)

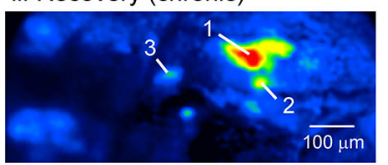

iii. Recovery (acute)

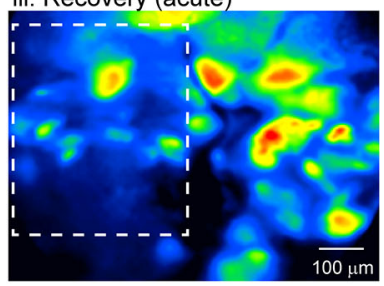

D

Pre exposure

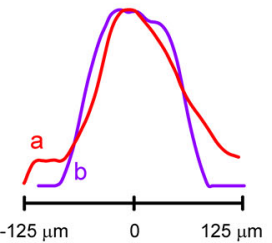

Recovery

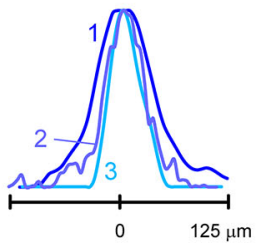

B Single odorant maps

i. Pre exposure

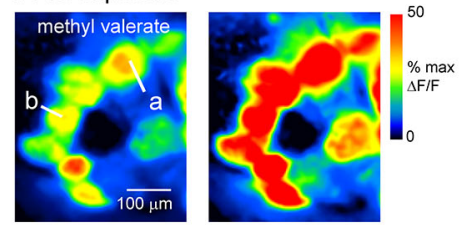

ii. Recovery (acute)

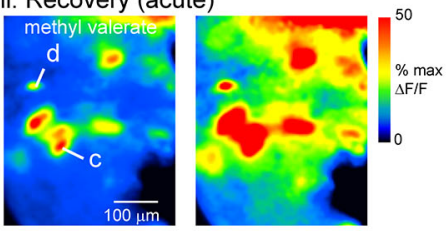

C

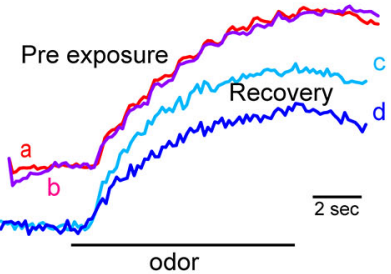

E

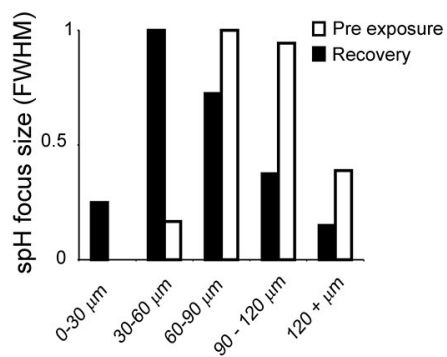

FIGURE 5 | Evidence for atypical convergence of OSNs onto OB targets after recovery from $\mathrm{MeBr}$ lesion. (A) Composite odorant response maps imaged at higher-magnification (20X objective) in unlesioned (i) and lesion-recovered (ii, iii) OBs. Maps are maximal-value projections of responses to all odorants tested in a given session (see Section Materials and Methods). In both chronically-imaged (ii) and acutely-imaged (iii) mice, odorants evoked $\mathrm{spH}$ signal foci that were smaller in size than a typical glomerulus. Boxes in (i and $\mathbf{i i i )}$ indicate regions rescaled in (B). (B) Response maps from the regions in (A) evoked by a single odorant (methyl valerate, $1 \%$ s.v.), scaled to their own maximum (left) and to $50 \%$ of their maximum (right) to highlight weaker-activated regions for both Pre-exposure (i) and Recovery conditions (ii). Smaller-sized foci are still apparent after rescaling. (C) Time-course of spH signal in typical and undersized foci in unlesioned (a, b) and lesion-recovered (c, d) animals. Traces taken from locations indicated in (B). Unlesioned and lesion-recovered traces are offset and scaled separately to compare signal time-course. (D) Normalized intensity profiles through $\mathrm{spH}$ foci taken from response maps in unlesioned $(\mathrm{a}, \mathrm{b})$ and lesion-recovered animals (1, 2, 3; see $\mathbf{A}$, ii), indicating smaller focus size in recovered animals. (E) Histogram of $\mathrm{spH}$ focus sizes for pre-exposure and lesion-recovered preparations. Lesion-recovered animals show an increase in the number of foci below $60 \mu \mathrm{m}$ full-width at half-maximum (FWHM). Bin size of the histogram $(20 \mu \mathrm{m})$ is 1 standard deviation of the FWHM values for pre-lesion OBs. the protected side of unilaterally-lesioned animals, OSN axons formed large bundles that coalesced into well-defined glomeruli defined by discrete, roughly spherical areas of OSN axon terminals (Figure 6A). In contrast, in lesion-recovered OBs OSNs often converged onto smaller structures and glomerular boundaries appeared less well-defined (Figures 6B, C). Qualitatively similar results were seen in chronically- and acutely-imaged lesion-recovered animals. Finally, nearly all lesion-recovered OBs showed at least some regions of the dorsal $\mathrm{OB}$ with no clear $\mathrm{spH}$ fluorescence, indicating a lack of reinnervation by OSNs (Figures 6B, C). The $\mathrm{OE}$ of these preparations had undergone substantial, although incomplete, reconstitution of the OSN population (Figures 6D-F). For example, the chronically-imaged mouse shown in Figures 6B, E showed substantial regeneration of the OE but nonetheless had patches where the OE thickness was thinner than the contralateral, unlesioned side (Figure 6E). Similarly, in the acutely-imaged example shown in Figures 6C, F, a greater extent of the dorsal $\mathrm{OE}$ does not recover fully or at all (Figure 6F), consistent with the lesser degree of glomerular reinnervation that was observed in this animals (Figure 6C).

To directly compare $\mathrm{spH}$ signal foci with the underlying anatomical structure of OSN inputs in lesion-recovered animals, 

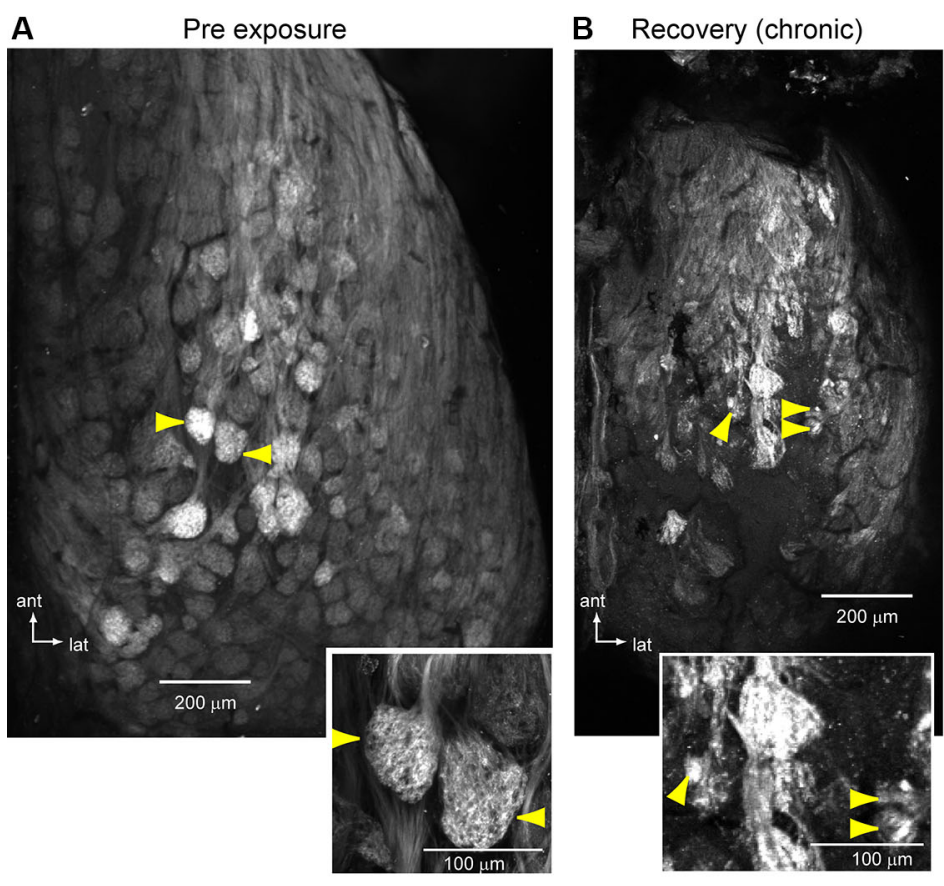

D

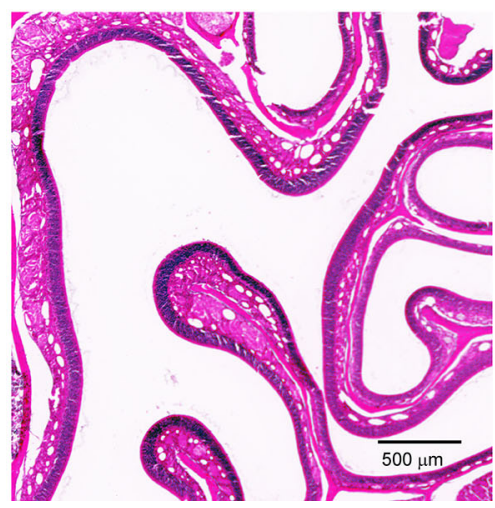

E Recovery (chronic)

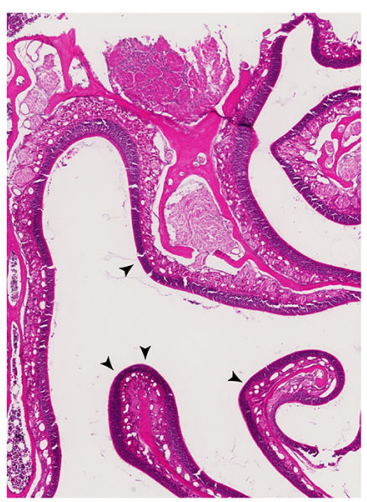

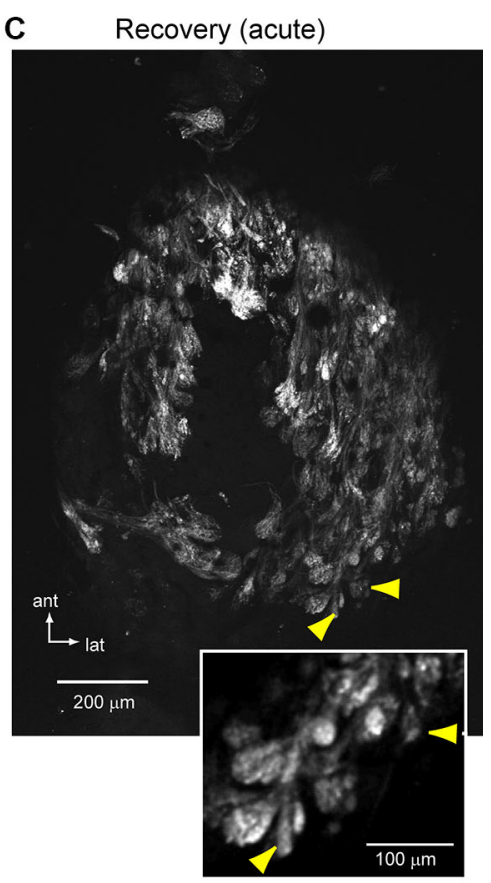

$\mathbf{F}$

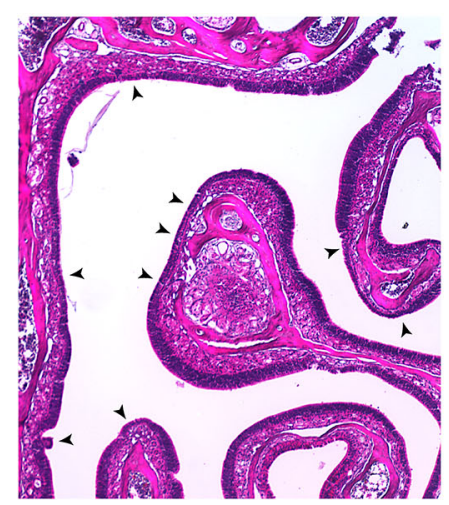

FIGURE 6 | Anatomical evidence for atypical convergence of OSNs onto OB glomeruli. (A) Confocal scan of a representative unlesioned OB (maximal $z$-stack projection). Inset shows detail of the glomeruli noted by yellow arrows. Glomeruli are clearly delineated and relatively uniform in size. (B, C) Confocal scans from two lesion-recovered animals, one chronically-imaged (B) and one acutely imaged (C). In both cases, glomerular borders are less distinct and OSNs often terminate in smaller structures (yellow arrowheads, detail in insets). In addition, regions of the central dorsal OB appear to lack innervation by OSNs. (D-F) H\&E-stained sections of the OE from the same animals shown in (A-C). In (E), there is substantial but not complete recovery of the epithelium. Arrowheads indicate areas with a reduced contingent of neurons as compared to the protected side. In (F), much of the epithelium remains less than fully recovered. Arrowheads indicate areas that are grossly abnormal and completely lacking in neurons. we imaged $\mathrm{spH}$ signals using in vivo 2PLSM in a subset of preparations ( $n=3$ chronically-imaged, 3 acutely-imaged, and 2 unexposed animals). Figure 7A shows resting fluorescence and odorant-evoked response maps imaged with wide-field epifluorescence and with 2PLSM in an unlesioned animal. OSN innervation of distinct glomeruli is clearly resolved in vivo using 2PLSM, and odorant-evoked $\mathrm{spH}$ signals are readily detectable. Odorants evoke $\mathrm{spH}$ signals throughout the glomerulus but with hot spots in smaller domains within it (Figure 6A), in agreement with previous reports (Wachowiak et al., 2004).
spH signals imaged with 2PLSM in lesion-recovered animals revealed qualitatively different signals with respect to glomerular structure. OSN axons often converged to atypically small structures (Figure 7B) or failed to delineate glomeruli with clear boundaries (Figure 7C). In these areas odorants often evoked $\mathrm{spH}$ signals appearing as "hot-spots" that appeared in only a portion of the glomerular structure (Figure 7B). Nonetheless, different odorants evoked different spatial distributions of $\mathrm{spH}$ signals (Figure 7C), consistent with their activating distinct (although smaller) populations of convergent OSNs. Overall, these results 
A Pre exposure
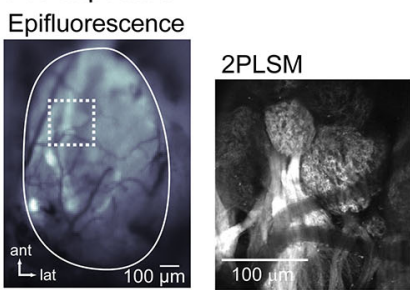

B Recovery 2PLSM
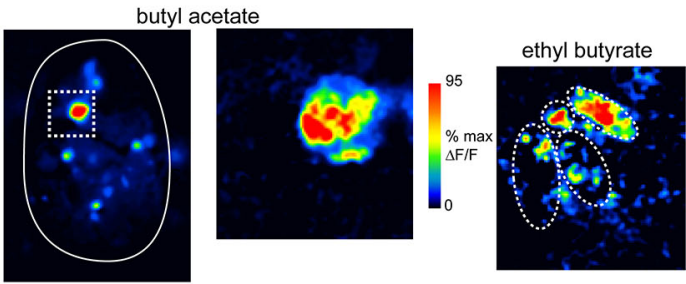

C Recovery

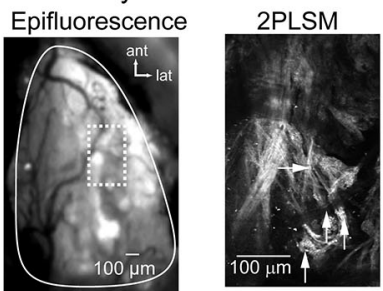

ethyl butyrate

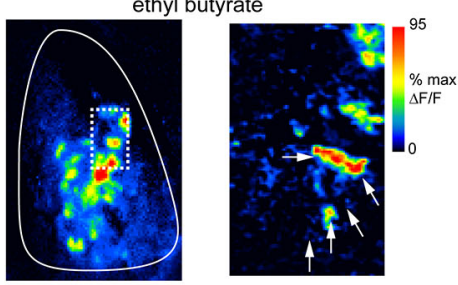

butyl acetate

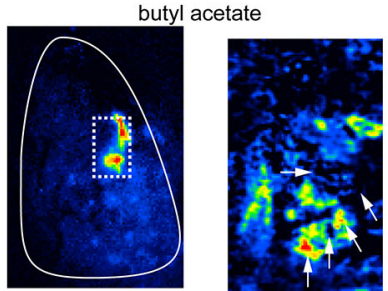

FIGURE 7 | Atypical glomerular convergence of OSNs confirmed with in vivo two-photon imaging of $\mathbf{s p H}$ signals. (A) Resting fluorescence and evoked $\mathrm{spH}$ response maps imaged with wide-field epifluorescence (left) and 2-photon laser scanning microscopy (2PLSM; right) in the same unlesioned animal. Epifluorescence image taken with $4 x$ objective. Odorant, butyl acetate. Dashed box indicates area imaged at higher-magnification with 2PLSM. With 2PLSM, spH fluorescence increases are apparent throughout the glomerulus, with "hot-spots" of high signal amplitude in subglomerular regions. (B) 2PLSM resting fluorescence (top) and response map (bottom) from a lesion-recovered animal.

Glomerular boundaries are less well-defined (compare to $(\mathbf{A})$ ); in this example several relatively discrete structures are apparent (indicated by dashed ovals), one of which is only 30-40 $\mu \mathrm{m}$ in diameter (top). Response map (bottom) shows spH signals with approximate boundaries of the four structures overlaid. In two of these structures, odorant (ethyl butyrate, 5\% s.v.) only evokes signals in a few foci, with the rest of the area showing no response. (C) Low-magnification wide-field (left) and high-magnification 2PLSM imaging (right) from another lesion-recovered animal, showing odorant-specific distribution of $\mathrm{spH}$ signals lacking a clear glomerular

\section{FIGURE 7 | Continued}

structure. Top: Resting fluorescence of the imaged regions. 2PLSM image is a projection of a z-stack through the olfactory nerve and glomerular layers. Middle, bottom: response maps evoked by ethyl butyrate and butyl acetate. Epifluorescence maps are unsmoothed (unlike previous figures). $\mathrm{spH}$ signals imaged with 2PLSM from the regions containing the strongest responses to both odorants (dashed box) reveal no clear glomerular structure from resting fluorescence. Instead, signals are distributed in small "hot-spots" (white arrows) whose distribution differs for the two odorants.

suggest that the smaller-sized $\mathrm{spH}$ signal foci observed in lesionrecovered OBs reflect OSN axon projections that do not converge to a canonical glomerular structure but which nonetheless provide functional input to $\mathrm{OB}$ targets.

\section{FUNCTIONAL RECOVERY OF OLFACTORY SENSORY NEURON (OSN) INPUTS AFTER SEVERE AND LASTING DAMAGE TO THE OE}

Exposure to higher doses of $\mathrm{MeBr}$ can lead to more pronounced damage to the OE that allows for only a limited recovery and regeneration of OSNs (Schwob et al., 1995, 1999). To test the limits at which OSNs can recover functional connections to the $\mathrm{OB}$, we unilaterally exposed an additional six animals to a higher MeBr dosage (240 ppm, $8 \mathrm{~h}$ ). This dosage was lethal in all but three animals. In these animals, $\mathrm{spH}$ signals were imaged in a single, acute session after the 12 week recovery period.

Confocal scans of the dorsal $\mathrm{OB}$ of these animals showed reduced resting $\mathrm{spH}$ fluorescence and no clear glomerular structure (Figure 8A); the OB on the protected side appeared normal. Histological examination of the $\mathrm{OE}$ of these animals showed extensive and lasting damage on the exposed side, such that there was little reconstitution of the neuronal population (Figure 8B). Instead, the majority of the epithelium had undergone respiratory metaplasia, in which damaged $\mathrm{OE}$ is replaced by respiratory epithelium after destruction of globose basal cells by severe MeBr exposure (Schwob et al., 1995; Jang et al., 2003). In these cases, the architecture of the epithelium and underlying lamina propria is distorted by fibrosis within what was the nasal cavity and the formation of synechiae bridging across the cavity from turbinate to septum (Figure 8B). In all three mice, this type of scarring was more prevalent in the anterior nasal cavity.

In vivo, the $\mathrm{OB}$ on the lesioned side of all three animals showed greatly reduced resting $\mathrm{spH}$ fluorescence (mean \pm s.d., lesion: $4153 \pm 516$ arbitrary fluorescence units, unexposed: 8884 $\pm 981, n=3$ animals), indicative of poor regeneration of OSN inputs (Figure $8 \mathrm{C}$ ). In addition, odorant-evoked $\mathrm{spH}$ signals were severely attenuated. However, in each of the animals at least some odorants evoked weak but detectable $\mathrm{spH}$ signals; in all cases these were confined to the lateral margins of the dorsal OB (Figures 8C, D). Higher-magnification (20x) imaging of this region revealed numerous small $\mathrm{spH}$ signal foci or diffuse, nonfocal signals. Despite the small amplitudes and greatly perturbed spatial organization of $\mathrm{spH}$ signals in these animals, different odorants still evoked spatially distinct response patterns (Figure 8D). These results indicate that at least some OSNs are capable of regenerating and reestablishing odorant-specific 
A Severe lesion (recovery, $\mathrm{OB}$ ) Exposed

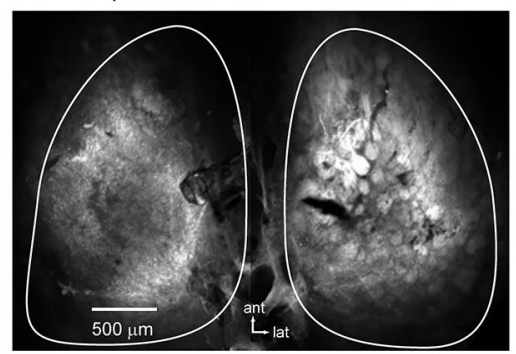

B Severe lesion (recovery, OE)

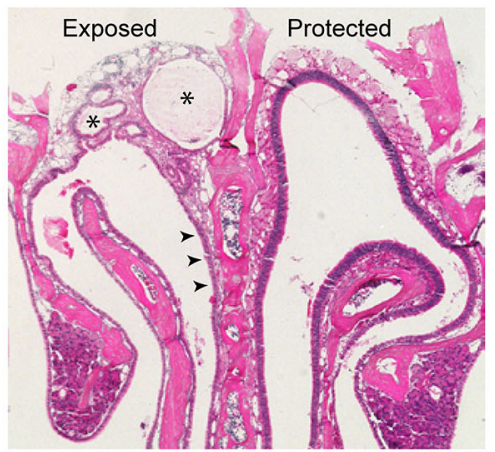

C Severe lesion (recovery) Exposed
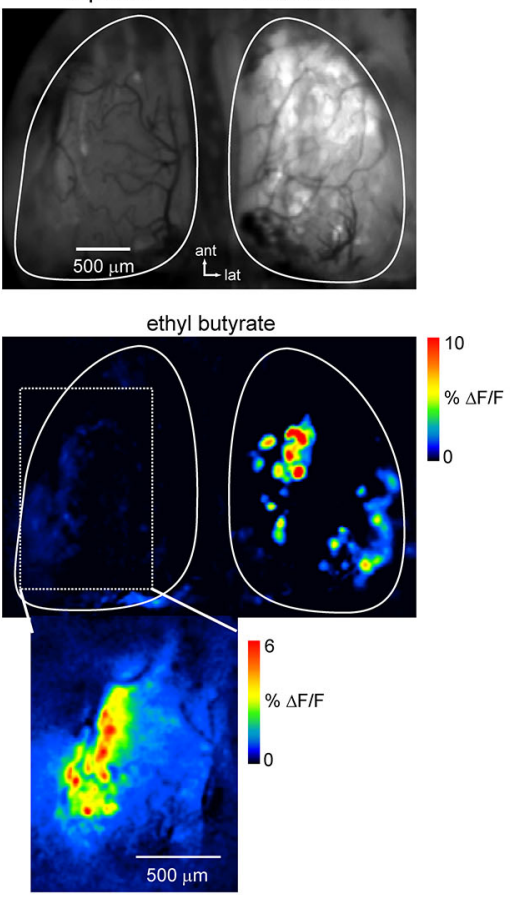

D Epifl., 20x mag

ethyl butyrate

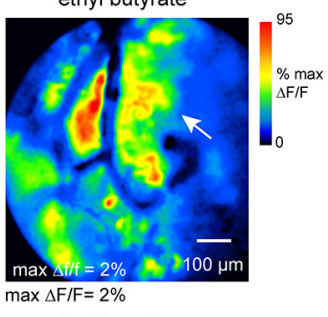

butyl acetate
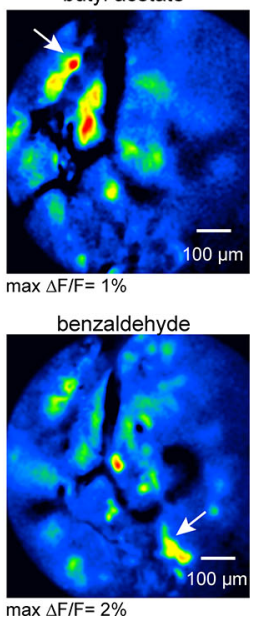

FIGURE 8 | OSNs can partially reestablish functional inputs to the $O B$ after severe and lasting trauma to the olfactory epithelium. (A) Confocal scan (maximal projection) of the dorsal OBs of an animal imaged at recovery stage after high-dosage unilateral $\mathrm{MeBr}$ exposure (See the text). The OB on the protected side appears normal; the $\mathrm{OB}$ on the exposed side fails to show OSNs terminating in glomerular structures. (B) H\&E-stained section of OE from the animal shown in (A). On the lesioned side there is almost no reconstitution of the neuronal population, although neurons are apparent in some areas of the epithelium (arrowheads). Scar tissue fills much of the dorsal meatus in this animal (asterisks). (C) Epifluorescence image and $\mathrm{spH}$ response map from the same animal as in
(A, B) imaged at the recovery stage. Resting fluorescence is low on the exposed side. Very weak odorant-evoked spH signals were detected on this side and were confined to the lateral margin of the dorsal OB (bottom). Inset shows evoked signals from this region after rotating the head for improved optical access and scaling responses in this region to their own maximum. (D) Response maps evoked by ethyl butyrate and two additional odorants imaged from the same animal at

higher-magnification (20X objective) with wide-field optics. Multiple spH signal foci, nearly all of which are subglomerular in size, are evoked by each odorant, although the patterns of activation are distinct. Arrows indicate signal foci that appear specific for a given odorant. functional connections with the $\mathrm{OB}$ even in the face of severe and lasting damage to the $\mathrm{OE}$.

\section{DISCUSSION}

We assessed the capacity of the mammalian olfactory system to reestablish functional connections to the CNS and to recapitulate odor representations at the level of the OB after wholesale destruction of the OSN population. By imaging odorant-evoked $\mathrm{spH}$ signals from OSNs to OB glomeruli before peripheral lesion and after a 12 week recovery period, we found that this regenerative capacity is robust: odor "maps" involving many glomeruli (and thus many ORs) were reconstituted with little or no change in their topographic organization across the dorsal OB. We also obtained evidence that mistargeted OSNs - which have previously only been observed anatomically-make functional connections to the OB. Finally, we found that OSNs were able to at least partially reestablish functional connections to the $\mathrm{OB}$ even after lesions severe enough to permit only minor recovery of the OSN population. These results expand on earlier anatomical studies that have reported regeneration and glomerular convergence of a few OR- and histologically-defined OSN populations (Schwob et al., 1999; Costanzo, 2000; St. John and Key, 2003; McMillan Carr et al., 2004; Blanco-Hernández et al., 2012) and are consistent with a recent report that discriminative odor memories are preserved after OSN lesion and recovery (Blanco-Hernández et al., 2012).

Several lines of evidence suggest that the process of installing a chronic imaging window did not substantially affect OSN targeting. First, in unlesioned (but windowed) controls, we found that odor maps remained stable for at least 13 and for as long as 25 weeks. Second, in unilaterally-lesioned animals, we observed differences in the fine structure of response maps (described below) between the MeBr-exposed and protected sides, despite the presence of an imaging window on each side. Third, we obtained qualitatively and quantitatively similar results in animals imaged only at the 12 week recovery time-point and exposed 
bilaterally to MeBr. Thus, it is unlikely that the chronic imaging procedure or unilateral lesion affected the process of OSN regeneration and targeting to glomeruli.

\section{RECOVERY OF ODOR MAP TOPOGRAPHY AFTER LESION}

In the mammalian $\mathrm{OB}$, many odorants preferentially evoke activity in glomeruli clustered in spatial domains covering several hundred microns (Imamura et al., 1992; Uchida et al., 2000; Johnson et al., 2002). These domains are associated with odorants of a particular chemical class and are innervated by molecularly and functionally distinct OSN types (Bozza et al., 2004, 2009; Takahashi et al., 2004; Matsumoto et al., 2010; Pacifico et al., 2012). We found that this domain organization, as assessed functionally across the dorsal $\mathrm{OB}$, is largely preserved after $\mathrm{OE}$ regeneration. This result does not simply reflect the reconstitution of normal zones of odorant receptor (OR) expression in the OE and the maintenance of rhinotopic projections from the $\mathrm{OE}$ to OB (Schoenfeld et al., 1994; Cummings et al., 2000; Iwema et al., 2004), as OSNs projecting to OB domains are interspersed in the OE (Bozza et al., 2009). The reconstitution of functional topography after OSN regeneration is consistent with earlier anatomical studies examining the targeting of P2- or M72expressing OSNs or of immunohistochemically-defined OSN subsets (Cummings et al., 2000; St. John and Key, 2003; McMillan Carr et al., 2004; Blanco-Hernández et al., 2012); these studies found that OSNs project to glomeruli in topographically similar locations as in control animals, although with clear errors in targeting. The fact that, in this study, spH response maps-even those involving many glomeruli-retain a spatial organization that matches that before lesion suggests that regenerated OSN axons do not randomly converge onto $\mathrm{OB}$ glomeruli but instead preferentially target their appropriate domain on the OB surface. In addition we note that many lesion-recovered response maps included individual signal foci (i.e., glomeruli) that appeared in a similar location to that observed before lesion (e.g., Figure 4A) or to that of a focus on the unexposed side (e.g., Figure 3D), suggesting that the precision of targeting in regenerated OSNs may be higher than of a functional domain.

The mechanisms mediating this targeting remain unclear but may include OR identity (Feinstein et al., 2004), OSN cell type (Bozza et al., 2009) and axon guidance cues (Schwob, 2002; Schwarting and Henion, 2011). In many systems such guidance mechanisms function only during a restricted developmental window; our results suggest that they are at least partially effective in guiding OSN axons in adults. The precise targeting of OSNs to glomeruli may also be affected by activity-dependent mechanisms driven by exposure to ambient odorants (Nakatani et al., 2003; Zou et al., 2004; Kerr and Belluscio, 2006). Examining the recovery of OSNs expressing markers for ORs for which ligands are known in combination with functional imaging of inputs to all glomeruli will be useful for testing this possibility.

It remains possible that a minority of OSNs expressing the same OR fail to converge onto glomeruli in their appropriate domains, or that a minority of OSN populations converge onto glomeruli in inappropriate locations. Quantifying the degree to which such mistargeting occurs is difficult since functional domains can only be loosely defined by odorant responsiveness
(Wachowiak and Cohen, 2001; Bozza et al., 2004; Wachowiak et al., 2013) (i.e., nearly all odorants activate at least some OSN input to glomeruli outside of their preferred domain) and because molecular (e.g., OR-based) tags to define domain boundaries are themselves derived from OSN convergence patterns (Bozza et al., 2009; Pacifico et al., 2012). It is also possible that $\mathrm{spH}$ signal foci that occur in similar locations before and after lesion may reflect activation of OSNs that express different ORs but have a similar odorant-specificity. An ideal approach to more precisely define the precision of OSN retargeting would be to tag the postsynaptic neurons associated with a given OSN populationfor example, using transysnaptic transgene expression driven by OSNs expressing a particular OR.

\section{OLFACTORY SENSORY NEURON (OSN) CONVERGENCE ONTO INDIVIDUAL GLOMERULI}

Another fundamental feature of OSN projections to the $\mathrm{OB}$ is the exclusive convergence of OSNs expressing the same OR onto a glomerulus (Mombaerts et al., 1996; Treloar et al., 2002). We found evidence for errors in this convergence after OE regeneration: lesion-recovered animals showed an increase in the number of small-sized $(<60 \mu \mathrm{m}) \mathrm{spH}$ foci compared to controls, indicating either reduced numbers of OSN axons forming a glomerulus or partial innervation of a glomerulus by OSNs expressing a given OR. We found evidence for both possibilities using high-resolution two-photon imaging of $\mathrm{spH}$ signals in register with the underlying structure of OSN axons. The time-course and odorant-specificity of $\mathrm{spH}$ signals in these subglomerular foci was similar to $\mathrm{spH}$ signals in larger foci or in unlesioned controls, suggesting that transmitter release from these OSN terminals was functional. These results suggest that regenerated OSNs can provide functional input to mistargeted glomeruli and that this mistargeting is a general phenomenon seen across many OR types.

OSN mistargeting may impact odor perception after OE lesion and recovery (Yee and Costanzo, 1998; Vedin et al., 2004). In humans, dysosmias are often reported after trauma to the OE (Doty, 1979; Meisami et al., 1998). Innervation of a single glomerulus by multiple OSN types might underlie these effects. Given our evidence that OSN mistargeting is restricted to within a functional $\mathrm{OB}$ domain, one prediction is that discrimination of structurally similar odorants will be impaired after OE lesion and recovery, while a second is that discrimination between odorants activating different domains will be unaffected. The former prediction has not, to our knowledge, been tested. The latter prediction is supported by a recent report that behavioral discriminations of two odorants that activate distinct $\mathrm{OB}$ domains persist after OSN lesion and recovery (Blanco-Hernández et al., 2012). However, a second study found that odor discriminations are impaired even after partial lesion that spares many OSNs (Bracey et al., 2013), in apparent contradiction to the recovery of odor memories after lesion and regeneration. Thus, rigorously testing perceptual effects of OSN mistargeting may be difficult and will likely require combining multiple perceptual assays with imaging of odor maps in the same animals (Bracey et al., 2013). 


\section{FACTORS AFFECTING OLFACTORY SENSORY NEURON (OSN) REGENERATION AND AXON TARGETING}

While we evaluated the recovery of sensory inputs to the $\mathrm{OB}$ after $\mathrm{MeBr}$-induced OSN lesion, previous studies have used a variety of lesion models with qualitatively distinct results. Severing the olfactory nerve at the cribriform plate, which leads to extensive OSN death and subsequent regeneration, results in more extensive mistargeting after regeneration-including a loss of rhinotopic projections - than does chemically lesioning OSNs (Costanzo, 2000; Christensen et al., 2001). There also appears to be a correlation between the numbers of OSNs surviving the lesion and the degree of mistargeting (Schwob et al., 1999). For example, retargeting of $\mathrm{P} 2$-expressing OSNs to their appropriate glomerulus is normal if these neurons alone are selectively lesioned using a genetic method while all other OSNs are spared (Gogos et al., 2000), and chemical lesions that spare the lamina propria appear to permit more precise targeting of recovered OSNs (BlancoHernández et al., 2012).

After severe $\mathrm{OE}$ lesion with higher $\mathrm{MeBr}$ doses in which there was substantial lasting damage to the $\mathrm{OE}$, we found that odor response maps were severely disrupted, with little or no regeneration of OSN inputs to the dorsal OB and a lack of convergence onto clear glomeruli in the lateral OB. Despite this severe disruption, however, odorants did evoke $\mathrm{spH}$ signals in reinnervated areas and did so with odorant-specific (though poorly-defined) spatial patterns, indicating that OSNs are capable of establishing functional inputs to the $\mathrm{OB}$ even in the absence of glomerular convergence. Thus the capacity of the OE to reestablish connections to the CNS appears to persist even in the face of extreme peripheral damage.

\section{AUTHOR CONTRIBUTIONS}

Man C. Cheung, Matt Wachowiak and James E. Schwob designed the experiments, Man C. Cheung performed the imaging experiments and data analysis, James E. Schwob administered the MeBr lesions, James E. Schwob and Woochan Jang performed the histological analysis and Matt Wachowiak and Man C. Cheung wrote the paper.

\section{ACKNOWLEDGMENTS}

We thank J. Mertz for providing scanning hardware and software for multiphoton microscopy, D. Wesson and J. McGann for assistance with naris occlusion experiments, T. Bozza for helpful advice and comments on the manuscript and M. Economo for assistance with data analysis. This work was supported by funding from the NIH (DC008116 and ARRA supplement 008116S1).

\section{REFERENCES}

Blanco-Hernández, E., Valle-Leija, P., Zomosa-Signoret, V., Drucker-Colín, R., and Vidaltamayo, R. (2012). Odor memory stability after reinnervation of the olfactory bulb. PLoS One 7:e46338. doi: 10.1371/journal.pone.0046338

Bozza, T., McGann, J. P., Mombaerts, P., and Wachowiak, M. (2004). In vivo imaging of neuronal activity by targeted expression of a genetically encoded probe in the mouse. Neuron 42, 9-21. doi: 10.1016/s0896-6273(04)00144-8

Bozza, T., Vassalli, A., Fuss, S., Zhang, J. J., Weiland, B., Pacifico, R., et al. (2009). Mapping of class I and class II odorant receptors to glomerular domains by two distinct types of olfactory sensory neurons in the mouse. Neuron 61, 220-233. doi: 10.1016/j.neuron.2008.11.010
Bracey, E. F., Pichler, B., Schaefer, A. T., Wallace, D. J., and Margrie, T. W. (2013). Perceptual judgements and chronic imaging of altered odour maps indicate comprehensive stimulus template matching in olfaction. Nat. Commun. 4:2100. doi: $10.1038 /$ ncomms3100

Chen, X., Fang, H., and Schwob, J. E. (2004). Multipotency of purified, transplanted globose basal cells in olfactory epithelium. J. Comp. Neurol. 469, 457-474. doi: 10.1002/cne.11031

Christensen, M. D., Holbrook, E. H., Costanzo, R. M., and Schwob, J. E. (2001). Rhinotopy is disrupted during the re-innervation of the olfactory bulb that follows transection of the olfactory nerve. Chem. Senses 26, 359-369. doi: 10. 1093/chemse/26.4.359

Costanzo, R. M. (2000). Rewiring the olfactory bulb: changes in odor maps following recovery from nerve transection. Chem. Senses 25, 199-205. doi: 10. 1093/chemse/25.2.199

Costanzo, R. M., and Kobayashi, M. (2010). Age-related changes in P2 odorant receptor mapping in the olfactory bulb. Chem. Senses 35, 417-426. doi: 10. 1093/chemse/bjq029

Cummings, D. M., Emge, D. K., Small, S. L., and Margolis, F. L. (2000). Pattern of olfactory bulb innervation returns after recovery from reversible peripheral deafferentation. J. Comp. Neurol. 421, 362-373. doi: 10.1002/(sici)10969861(20000605)421:3<362::aid-cne5>3.0.co;2-8

Cummings, D. M., Henning, H. E., and Brunjes, P. C. (1997). Olfactory bulb recovery after early sensory deprivation. J. Neurosci. 17, 7433-7440.

Doty, R. L. (1979). A review of olfactory dysfunctions in man. Am. J. Otolaryngol. 1, 57-79. doi: 10.1016/s0196-0709(79)80010-1

Feinstein, P., Bozza, T., Rodriguez, I., Vassalli, A., and Mombaerts, P. (2004). Axon guidance of mouse olfactory sensory neurons by odorant receptors and the beta2 adrenergic receptor. Cell 117, 833-846. doi: 10.1016/j.cell.2004.05.013

Gogos, J. A., Osborne, J., Nemes, A., Mendelsohn, M., and Axel, R. (2000). Genetic ablation and restoration of the olfactory topographic map. Cell 103, 609-620. doi: 10.1016/s0092-8674(00)00164-1

Imamura, K., Mataga, N., and Mori, K. (1992). Coding of odor molecules by mitral/tufted cells in rabbit olfactory bulb. I. Aliphatic compounds. J. Neurophysiol. 68, 1986-2002.

Iwema, C. L., Fang, H., Kurtz, D. B., Youngentob, S. L., and Schwob, J. E. (2004). Odorant receptor expression patterns are restored in lesion-recovered rat olfactory epithelium. J. Neurosci. 24, 356-369. doi: 10.1523/jneurosci.121903.2004

Jang, W., Youngentob, S. L., and Schwob, J. E. (2003). Globose basal cells are required for reconstitution of olfactory epithelium after methyl bromide lesion. J. Comp. Neurol. 460, 123-140. doi: 10.1002/cne.10642

Johnson, B. A., Ho, S. L., Xu, Z., Yihan, J. S., Yip, S., Hingco, E. E., et al. (2002). Functional mapping of the rat olfactory bulb using diverse odorants reveals modular responses to functional groups and hydrocarbon structural features. J. Comp. Neurol. 449, 180-194. doi: 10.1002/cne.10284

Jones, S. V., Choi, D. C., Davis, M., and Ressler, K. J. (2008). Learning-dependent structural plasticity in the adult olfactory pathway. J. Neurosci. 28, 13106-13111. doi: 10.1523/jneurosci.4465-08.2008

Kass, M. D., Moberly, A. H., Rosenthal, M. C., Guang, S. A., and McGann, J. P. (2013). Odor-specific, olfactory marker protein-mediated sparsening of primary olfactory input to the brain after odor exposure. J. Neurosci. 33, 6594-6602. doi: 10.1523/jneurosci.1442-12.2013

Kerr, M. A., and Belluscio, L. (2006). Olfactory experience accelerates glomerular refinement in the mammalian olfactory bulb. Nat. Neurosci. 9, 484-486. doi: 10. $1038 / \mathrm{nn} 1673$

Lam, Y.-W., Cohen, L. B., Wachowiak, M., and Zochowski, M. R. (2000). Odors elicit three different oscillations in the turtle olfactory bulb. J. Neurosci. 20, 749762.

Matsumoto, H., Kobayakawa, K., Kobayakawa, R., Tashiro, T., Mori, K., and Sakano, H. (2010). Spatial arrangement of glomerular molecular-feature clusters in the odorant-receptor class domains of the mouse olfactory bulb. $J$. Neurophysiol. 103, 3490-3500. doi: 10.1152/jn.00035.2010

McGann, J. P., Pírez, N., Gainey, M. A., Muratore, C., Elias, A. S., and Wachowiak, M. (2005). Odorant representations are modulated by intra- but not interglomerular presynaptic inhibition of olfactory sensory neurons. Neuron 48, 1039-1053. doi: 10.1016/j.neuron.2005.10.031

McMillan Carr, V., Ring, G., Youngentob, S. L., Schwob, J. E., and Farbman, A. I. (2004). Altered epithelial density and expansion of bulbar projections of a discrete HSP70 immunoreactive subpopulation of rat olfactory receptor 
neurons in reconstituting olfactory epithelium following exposure to methyl bromide. J. Comp. Neurol. 469, 475-493. doi: 10.1002/cne.11020

Meisami, E., Mikhail, L., Baim, D., and Bhatnagar, K. P. (1998). Human olfactory bulb: aging of glomeruli and mitral cells and a search for the accessory olfactory bulb. Ann. N Y Acad. Sci. 855, 708-715. doi: 10.1111/j.1749-6632.1998.tb10 649.x

Meister, M., and Bonhoeffer, T. (2001). Tuning and topography in an odor map on the rat olfactory bulb. J. Neurosci. 21, 1351-1360.

Mombaerts, P., Wang, F., Dulac, C., Chao, S. K., Nemes, A., Mendelsohn, M., et al. (1996). Visualizing an olfactory sensory map. Cell 87, 675-686. doi: 10. 1016/s0092-8674(00)81387-2

Nagao, H., Yamaguchi, M., Takahash, Y., and Mori, K. (2002). Grouping and representation of odorant receptors in domains of the olfactory bulb sensory map. Microsc. Res. Tech. 58, 168-175. doi: 10.1002/jemt.10146

Nakatani, H., Serizawa, S., Nakajima, M., Imai, T., and Sakano, H. (2003). Developmental elimination of ectopic projection sites for the transgenic OR gene that has lost zone specificity in the olfactory epithelium. Eur. J. Neurosci. 18, 2425 2432. doi: 10.1046/j.1460-9568.2003.02998.x

Oka, Y., Takai, Y., and Touhara, K. (2009). Nasal airflow rate affects the sensitivity and pattern of glomerular odorant responses in the mouse olfactory bulb. $J$. Neurosci. 29, 12070-12078. doi: 10.1523/jneurosci.1415-09.2009

Pacifico, R., Dewan, A., Cawley, D., Guo, C., and Bozza, T. (2012). An olfactory subsystem that mediates high-sensitivity detection of volatile amines. Cell Rep. 2, 76-88. doi: 10.1016/j.celrep.2012.06.006

Pírez, N., and Wachowiak, M. (2008). In vivo modulation of sensory input to the olfactory bulb by tonic and activity-dependent presynaptic inhibition of receptor neurons. J. Neurosci. 28, 6360-6371. doi: 10.1523/jneurosci.0793-08. 2008

Schaefer, M. L., Finger, T. E., and Restrepo, D. (2001). Variability of position of the P2 glomerulus within a map of the mouse olfactory bulb. J. Comp. Neurol. 436, 351-362. doi: 10.1002/cne.1072

Schoenfeld, T. A., Clancy, A. N., Forbes, W. B., and Macrides, F. (1994). The spatial organization of the peripheral olfactory system of the hamster. part I: receptor neuron projections to the main olfactory bulb. Brain Res. Bull. 34, 183-210. doi: 10.1016/0361-9230(94)90059-0

Schwarting, G. A., and Henion, T. R. (2011). Regulation and function of axon guidance and adhesion molecules during olfactory map formation. J. Cell. Biochem. 112, 2663-2671. doi: 10.1002/jcb.23203

Schwob, J. E. (2002). Neural regeneration and the peripheral olfactory system. Anat. Rec. 269, 33-49. doi: 10.1002/ar.10047

Schwob, J. E., and Gottlieb, D. I. (1986). The primary olfactory projection has two chemically distinct zones. J. Neurosci. 6, 3393-3404.

Schwob, J. E., Youngentob, S. L., and Mezza, R. C. (1995). Reconstitution of the rat olfactory epithelium after methyl bromide-induced lesion. J. Comp. Neurol. 359, 15-37. doi: 10.1002/cne.903590103

Schwob, J. E., Youngentob, S. L., Ring, G., Iwema, C. L., and Mezza, R. C. (1999). Reinnervation of the rat olfactory bulb after methyl bromide-induced lesion: timing and extent of reinnervation. J. Comp. Neurol. 412, 439-457. doi: 10. 1002/(sici) 1096-9861(19990927)412:3<439::aid-cne5>3.0.co;2-h

St. John, J., and Key, B. (2003). Axon mis-targeting in the olfactory bulb during regeneration of olfactory neuroepithelium. Chem. Senses 28, 773-779. doi: 10. 1093/chemse/bjg068
Strotmann, J., Conzelmann, S., Beck, A., Feinstein, P., Breer, H., and Mombaerts, P. (2000). Local permutations in the glomerular array of the mouse olfactory bulb. J. Neurosci. 20, 6927-6938.

Takahashi, Y. K., Kurosaki, M., Hirono, S., and Mori, K. (2004). Topographic representation of odorant molecular features in the rat olfactory bulb. J. Neurophysiol. 92, 2413-2427. doi: 10.1152/jn.00236.2004

Treloar, H. B., Feinstein, P., Mombaerts, P., and Greer, C. A. (2002). Specificity of glomerular targeting by olfactory sensory axons. J. Neurosci. 22, 2469-2477.

Uchida, N., Takahashi, Y. K., Tanifuji, M., and Mori, K. (2000). Odor maps in the mammalian olfactory bulb: domain organization and odorant structural features. Nat. Neurosci. 3, 1035-1043. doi: 10.1038/79857

Vedin, V., Slotnick, B., and Berghard, A. (2004). Zonal ablation of the olfactory sensory neuroepithelium of the mouse: effects on odorant detection. Eur. J. Neurosci. 20, 1858-1864. doi: 10.1111/j.1460-9568.2004.03634.x

Wachowiak, M., and Cohen, L. B. (2001). Representation of odorants by receptor neuron input to the mouse olfactory bulb. Neuron 32, 723-735. doi: 10 . 1016/s0896-6273(01)00506-2

Wachowiak, M., Denk, W., and Friedrich, R. W. (2004). Functional organization of sensory input to the olfactory bulb glomerulus analyzed by two-photon calcium imaging. Proc. Natl. Acad. Sci. U S A 101, 9097-9102. doi: 10.1073/pnas. 0400438101

Wachowiak, M., Economo, M. N., Díaz-Quesada, M., Brunert, D., Wesson, D. W., White, J. A., et al. (2013). Optical dissection of odor information processing in vivo using gcamps expressed in specified cell types of the olfactory bulb. $J$. Neurosci. 33, 5285-5300. doi: 10.1523/jneurosci.4824-12.2013

Xu, H. T., Pan, F., Yang, G., and Gan, W. B. (2007). Choice of cranial window type for in vivo imaging affects dendritic spine turnover in the cortex. Nat. Neurosci. 10, 549-551. doi: 10.1038/nn1883

Yee, K. K., and Costanzo, R. M. (1998). Changes in odor quality discrimination following recovery from olfactory nerve transection. Chem. Senses 23, 513-519. doi: $10.1093 /$ chemse/23.5.513

Zou, D.-J., Feinstein, P., Rivers, A. L., Mathews, G. A., Kim, A., Greer, C. A., et al. (2004). Postnatal refinement of peripheral olfactory projections. Science 304, 1976-1979. doi: 10.1126/science.1093468

Conflict of Interest Statement: The authors declare that the research was conducted in the absence of any commercial or financial relationships that could be construed as a potential conflict of interest.

Received: 09 October 2013; accepted: 20 December 2013; published online: 07 January 2014.

Citation: Cheung MC, Jang W, Schwob JE and Wachowiak M (2014) Functional recovery of odor representations in regenerated sensory inputs to the olfactory bulb. Front. Neural Circuits 7:207. doi: 10.3389/fncir.2013.00207

This article was submitted to the journal Frontiers in Neural Circuits.

Copyright (๑) 2014 Cheung, Jang, Schwob and Wachowiak. This is an open-access article distributed under the terms of the Creative Commons Attribution License (CC $B Y$ ). The use, distribution or reproduction in other forums is permitted, provided the original author(s) or licensor are credited and that the original publication in this journal is cited, in accordance with accepted academic practice. No use, distribution or reproduction is permitted which does not comply with these terms. 